\title{
Guidelines of Chinese Society of Clinical Oncology (CSCO) on Diagnosis and Treatment of Breast Cancer (2020 version)
}

\author{
Zefei Jiang ${ }^{1}$, Erwei Song ${ }^{2}$, Xiaojia Wang ${ }^{3}$, Haibo Wang ${ }^{4}$, Xiang Wang ${ }^{5}$, Jiong Wu ${ }^{6}$, Yongmei Yin ${ }^{7}$, \\ Qingyuan Zhang ${ }^{8}$, Jiayi Chen ${ }^{9}$, Wenyan Che ${ }^{10}$, Xiaoyuan Chen ${ }^{11}$, Zhanhong Chen ${ }^{3}$, Peifen Fu ${ }^{12}$, \\ Cuizhi Geng ${ }^{13}$, Baoliang Guo ${ }^{14}$, Chunfang Hao ${ }^{15}$, Yingjian $\mathrm{He}^{16}$, Feng Jin ${ }^{17}$, Ning Liao ${ }^{18}$, Nanlin $\mathrm{Li}^{19}$, \\ Jian $\mathrm{Liu}^{20}$, Qiang Liu ${ }^{2}$, Yunjiang Liu ${ }^{14}$, Yueping Liu ${ }^{14}$, Jie $\mathrm{Ma}^{21}$, Jianyun Nie ${ }^{22}$, Yueyin Pan ${ }^{23}$, \\ Chuangui Song ${ }^{24}$, Tao Sun ${ }^{25}$, Kun Wang ${ }^{19}$, Shu Wang ${ }^{26}$, Tao Wang ${ }^{1}$, Xin Wang ${ }^{5}$, Biyun Wang ${ }^{27}$, \\ Shusen Wang ${ }^{28}$, Yongsheng Wang ${ }^{29}$, Ling Xu ${ }^{30}$, Min Yan $^{31}$, Ying Yan ${ }^{17}$, Peng Yuan ${ }^{5}$, Junlan Yang ${ }^{32}$, \\ Fan Zhang ${ }^{32}$, Jun Zhang ${ }^{14}$, Shaohua Zhang ${ }^{1}$, Xuan Zeng ${ }^{33}$, Jianbin Li $^{34}$, Fengrui Xu ${ }^{1}$
}

${ }^{1}$ The Fifth Medical Center of Chinese PLA General Hospital, Beijing, China; ${ }^{2}$ Sun Yat-sen Memorial Hospital, Sun Yat-sen University, Guangzhou, China; ${ }^{3}$ Cancer Hospital Affiliated to the University of Chinese Academy of Sciences, Hangzhou, China; ${ }^{4}$ Affiliated Hospital of Qingdao University, Qingdao, China; ${ }^{5}$ Cancer Hospital \& Institute, Chinese Academy of Medical Sciences, Beijing, China; ${ }^{6}$ Cancer Hospital Affiliated to Fudan University, Shanghai, China; ${ }^{7}$ Jiangsu Provincial People's Hospital, Nanjing, China; ${ }^{8}$ Cancer Hospital Affiliated to Harbin Medical University, Harbin, China; ${ }^{9}$ Ruijin Hospital Affiliated to Shanghai Jiaotong University School of Medicine, Shanghai, China; ${ }^{10}$ the First Affiliated Hospital of Nanchang University, Nanchang, China; ${ }^{11}$ Tsinghua University School of Medicine, Beijing, China; ${ }^{12}$ the First Affiliated Hospital, Zhejiang University School of Medicine, Hangzhou, China; ${ }^{13}$ the Fourth Affiliated Hospital of Hebei Medical University, Shijiazhuang, China; ${ }^{14}$ the Second Affiliated Hospital of Harbin Medical University, Harbin, China; ${ }^{15}$ Tianjin Cancer Hospital, Tianjin, China; ${ }^{16}$ Tumor Hospital of Peking University, Beijing, China; ${ }^{17}$ the First Affiliated Hospital of China Medical University, Shenyang, China; ${ }^{18}$ Guangdong Provincial People's Hospital, Guangzhou, China; ${ }^{19}$ Air Force Medical University Affiliated Xijing Hospital, Xi'an, China; ${ }^{20}$ Fujian Provincial Tumor Hospital, Fuzhou, China; ${ }^{21}$ Tangshan People's Hospital, Tangshan, China; ${ }^{22}$ Yunnan Provincial Cancer Hospital, Kunming, China; ${ }^{23}$ Anhui Provincial Hospital, Hefei, China; ${ }^{24}$ Affiliated Union Hospital of Fujian Medical University, Fuzhou, China; ${ }^{25}$ Liaoning Provincial Cancer Hospital, Shenyang, China; ${ }^{26}$ People Hospital, Peking University, Beijing, China; ${ }^{27}$ Cancer Hospital Affiliated to Fudan University, Shanghai, China; ${ }^{28}$ Sun Yat-sen University Cancer Center, Guangzhou, China; ${ }^{29}$ Shandong Provincial Cancer Hospital, Jinan, China; ${ }^{30}$ the First Hospital of Peking University, Beijing, China; ${ }^{31}$ Henan Provincial Cancer Hospital, Shijiazhuang, China; ${ }^{32}$ the First Medical Center of the Chinese PLA General Hospital, Beijing, China; ${ }^{33}$ Peking Union Medical College Hospital, Beijing, China; ${ }^{34}$ Beijing Institute of Biotechnology, Beijing, China

Correspondence to: Zefei Jiang. Department of Breast Oncology, The Fifth Medical Center of Chinese PLA General Hospital, Beijing 100071, China. Email: jiangzefei@csco.org.cn.

Received: 10 August 2020; Accepted: 14 September 2020; Published: 31 October 2020.

doi: $10.21037 /$ tbcr-2020-2

View this article at: http://dx.doi.org/10.21037/tbcr-2020-2 


\section{Introduction}

Developing guidelines for the diagnosis and treatment of common cancers in China based on the evidence-based practice, the availability of diagnosis and treatment products, and the up-to-date advances in precision medicine is one of the basic tasks of the Chinese Society of Clinical Oncology (CSCO). In recent years, the availability of medical resources has become a major concern in clinical guidelines, which is particularly important for developing countries or socioeconomically diverse countries and territories. China is the world's largest developing country, with a large territory and uneven economic and academic developments. The CSCO guidelines must take into account the differences in regional development, the availability of medicines and diagnostic methods, and the social value of cancer treatment. Therefore, for each clinical problem and intervention in the CSCO guidelines, the levels of evidence should be graded according to the currently available evidences and expert consensuses, and the grades of recommendations should be based on the availability and cost-effectiveness of the products. Protocols with high evidence level and good availability are used as the Level I recommendations; protocols with relatively high evidence level but slightly lower expert consensus or with poor availability are used as the Level II recommendations; and protocols that are clinically applicable but with low evidence level are regarded as the Level III recommendations. Based on the findings of clinical research at home and abroad and the opinions of CSCO experts, the CSCO guidelines determine the levels of recommendations for clinical application. The CSCO Guidance Working Group firmly believes that evidence-based, availability-concerned, and consensus-based guidelines will be more feasible for clinical practice in China. Again, any comments from our readers are greatly appreciated and will be considered in updates of these guidelines, so as to maintain the accuracy, fairness, and timeliness of the CSCO guidelines.

\section{Preoperative neoadjuvant treatment of breast cancer}

\section{Indications for neoadjuvant treatment}

Patients who meet one of the following conditions may choose preoperative neoadjuvant treatment ${ }^{1}$ :

(I) Large tumor size $(>5 \mathrm{~cm})^{2}$;

(II) With positive axillary lymph nodes;

(III) HER-2-positive;

(IV) Triple-negative ${ }^{3}$;

(V) With a desire of breast conserving surgery, which, however, can not be achieved due to the proportion of tumor among breast.

Notes: ${ }^{1}$ Preoperative treatments includes chemotherapy, targeted therapy, and endocrine therapy. See the relevant sections in this part for more details.

${ }^{2}$ For primary breast masses sized $2.0-5.0 \mathrm{~cm}$, other biological markers should be tested before neoadjuvant treatment is performed.

${ }^{3}$ Most members of the CSCOBC expert group agree that when only "HER-2-positive" or "triple negative" is used as the criterion for neoadjuvant treatment of breast cancer, the tumor diameter should be larger than $2 \mathrm{~cm}$; or, the patient may participate in a rigorously-designed clinical trial.

\section{Preoperative treatment of HER-2-positive breast cancer ${ }^{1}$}

\begin{tabular}{|c|c|}
\hline Level I recommendations & Level II recommendations \\
\hline 1. $\mathrm{TCbHP}^{2}(1 \mathrm{~A})$ & $\begin{array}{l}\text { 1. Anti-HER-2 target therapy combined with taxane-based regimens }{ }^{4,5} \text { (2B) } \\
\text { such as } \mathrm{TCbH}(2 \mathrm{~A}) \text { and } \mathrm{AC} \rightarrow \mathrm{THP}(2 \mathrm{~B})\end{array}$ \\
\hline
\end{tabular}

Note: ${ }^{1}$ Clinical studies have proved that neoadjuvant treatment with trastuzumab plus chemotherapy significantly increase the pCR rate in HER-2-positive breast cancer patients, laying a foundation for trastuzumab as a standard agent in the neoadjuvant treatment of HER-2-positive breast cancer. The expert group generally recognizes that dual-targeted therapy 
can be considered during the neoadjuvant treatment in all patients who qualify for single-targeted therapy.

${ }^{2}$ The KRISTINE study (1) confirmed the effectiveness and safety of TCbHP regimen in neoadjuvant treatment, Therefore, $\mathrm{TCbHP}$ is the preferred regimen in preoperative treatment.

${ }^{3}$ The NeoSphere study (2) confirmed that adding pertuzumab to TH could further increase the pCR rate in HER-2-positive patients. However, in the above study, surgery was performed after 4 cycles of neoadjuvant treatment with THP, and the dualtargeted therapy was suspended after surgery and then continued after 3 cycles of FEC therapy. The clinical feasibility of this protocol was quite questionable.

${ }^{4}$ The $\mathrm{TCbH}$ regimen has been demonstrated to be effective and safe in preoperative neoadjuvant therapy and postoperative adjuvant therapy and thus can be recommended for neoadjuvant therapy (3).

${ }^{5} \mathrm{Based}$ on the findings of research on $\mathrm{AC} \rightarrow \mathrm{TH}$ regimen in the single-targeted era, some experts agree that $\mathrm{AC} \rightarrow \mathrm{THP}$ can be used as an optional regimen for neoadjuvant therapy, which, however, has not been validated in well-designed clinical studies. ${ }^{6}$ According to the GBG69 study (4), albumin paclitaxel has a higher pCR rate than solvent-based paclitaxel in neoadjuvant therapy and it can also improve patients' disease-free survival (DFS). Thus, albumin paclitaxel can also be an optional drug in neoadjuvant therapy.

\section{Adjuvant treatment for HER-2-positive patients after neoadjuvant therapy ${ }^{1}$}

As more HER-2-positive breast cancer patients receive neoadjuvant treatment, the strategies of postoperative adjuvant treatment have also changed. Accordingly, the 2020 version of these Guidelines recommends the following adjuvant treatments for HER-2-positive patients who have received neoadjuvant treatment:

\section{Preoperative anti-HER-2 treatment with trastuzumab alone}

\begin{tabular}{lll}
\hline Stratification & Level I recommendations & Level II recommendations \\
\hline Pathological complete response ( $\mathrm{pCR})^{2}$ & Trastuzumab $^{3}(1 \mathrm{~A})$ & HP (trastuzumab + pertuzumab) (2A) \\
Non-pathological complete response (nonpCR) & 1. HP (trastuzumab + pertuzumab) (2A) & Trastuzumab (2B) \\
& 2. T-DM1 (IB) & \\
\hline
\end{tabular}

\section{Preoperative anti-HER-2 treatment with HP}

\begin{tabular}{lll}
\hline Stratification & Level I recommendations & Level II recommendations \\
\hline Pathological complete response (pCR) & HP $^{3}$ (trastuzumab + pertuzumab) (1A) & Trastuzumab (2B) \\
Non-pathological complete response (nonpCR) & T-DM1 (1B) & HP (trastuzumab + pertuzumab) (2A) \\
\hline
\end{tabular}

Notes: ${ }^{1}$ The pre-planned treatment cycles must be completed during the neoadjuvant therapy for HER-2-positive breast cancer. Surgery is performed only after the full course of neoadjuvant therapy is completed. The postoperative adjuvant treatment is performed (or not) based on the implementation of neoadjuvant therapy and the achievement of postoperative pCR.

${ }^{2}$ Pathological evaluation is an important tool for evaluating the efficacy of pre-operative neoadjuvant chemotherapy and the pCR achieved after surgery. It is valuable for evaluating the effectiveness of neoadjuvant therapy and determining postoperative adjuvant treatment options.

${ }^{3}$ For patients who have reached pCR after a full course of neoadjuvant therapy, the initial targeted therapy should continue in the postoperative adjuvant therapy. For patients who only use trastuzumab before surgery, dual-targeted therapy can also be considered based on the outcomes of postoperative adjuvant therapy.

${ }^{4}$ Clinical studies have shown that the dual-targeted therapy with trastuzumab and pertuzumab is superior to trastuzumab alone. In the KATHERINE study (5), for patients who failed to achieve pCR after preoperative treatment with trastuzumab, adjuvant therapy with T-DM1 further improved the prognosis. Thus, T-DM1 can be considered for patients who fail to achieve pCR after preoperative anti-HER-2 treatment with trastuzumab alone. However, so far there is no definite evidence 
that T-DM1 is superior to HP therapy; in addition, the availability of T-DM1 drugs can also be questionable. Thus, in these Guidelines we prefer to use the $\mathrm{H}+\mathrm{P}$ regimen first. T-DM1 can be considered in patients who fail to achieve pCR after preoperative anti-HER-2 treatment with dual-targeted therapy.

\section{Preoperative chemotherapy of HER-2-negative breast cancer}

\begin{tabular}{ll}
\hline Level I recommendations & Level II recommendations \\
\hline $\begin{array}{l}\text { 1. Choose a regimen that contains both anthracyclines and } \\
\text { taxanes }{ }^{1}:\end{array}$ & 1. Other regimens based on anthracyclines and taxanes \\
$\operatorname{TAC}(1 \mathrm{~A})$ & $\mathrm{AC} \rightarrow \mathrm{T}(1 \mathrm{~B})$ \\
$\mathrm{AT}^{4,5}(2 \mathrm{~A})$ & $\begin{array}{l}\text { 2. For young, triple-negative patients, especially those with BRCA gene } \\
\text { mutation, regimens with both taxanes and platinum can be applied }\end{array}$ \\
& For example: TP (2A)
\end{tabular}

Note: ${ }^{1}$ The treatment regimen and cycles are decided before neoadjuvant chemotherapy according to different treatment aims. In principle, in patients who respond to the combination of anthracycline and taxanes, the neoadjuvant chemotherapy should be completed as planed, and the timing and procedure of surgery should be discussed timely. However, the chemotherapy regimen may be changed for those operable patients who respond poorly to the neoadjuvant chemotherapy. For instance, some patients may respond poorly to AT, in whom NP may be applied instead. If the response to sequential treatment is still poor, the treatment strategy should be adjusted and surgery should be performed in time. For triple-negative patients who do not reach pCR after completing preoperative treatment, 6 to 8 cycles of capecitabine should be given after surgery.

${ }^{2}$ Studies have shown that platinum can increase the pCR rate in patients with triple-negative breast cancer $(6,7)$. However, due to the lack of data from randomized controlled phase III trials, platinum-containing regimens are not routinely recommended for triple-negative breast cancer patients. Nevertheless, preoperative platinum-containing regimens can be applied in young, triple-negative patients, especially those with BRCA mutations.

${ }^{3}$ According to the KEYNOTE-522 study, adding a PD-L1 inhibitor to neoadjuvant chemotherapy increased the pCR rate in triple-negative breast cancer. Currently, TNBC patients are only recommended to participate in rigorously-designed trials.

${ }^{4}$ According to the GBG69 study (4), albumin paclitaxel has a higher pCR rate than solvent-based paclitaxel in neoadjuvant therapy and it can also improve patients' DFS.

${ }^{5} \mathrm{Few}$ evidences on the use of pirarubicin have been published in foreign literature. According to the experience in China, the expert group believes that pirarubicin can be considered in neoadjuvant treatment before surgery.

\section{Preoperative endocrine therapy for hormone receptor-positive breast cancer}

Preoperative endocrine therapy is feasible for the following populations: (I) patients who require preoperative treatment but are not suitable for chemotherapy; (II) patients who are temporarily unsuitable for surgery; and (III) hormone-dependent patients who do not need immediate surgery.

(I) For postmenopausal hormone receptor-positive patients, the aromatase inhibitors (including anastrozole, letrozole, and exemestane) are recommended for preoperative endocrine therapy; fulvestrant may be considered in some patients who are not suitable for aromatase inhibitors (e.g., with a bone density T-score of $<-2.5$ ). For premenopausal hormone receptor-positive patients, ovarian function suppression plus aromatase inhibitors may be applied. Some patients with locally advanced breast cancer requiring neoadjuvant endocrine therapy may be treated with endocrine therapy combined with CDK4/6 inhibitors or participate in clinical trials.

(II) Generally, the response to preoperative endocrine therapy should be evaluated every two months. If the treatment is effective and tolerable, it can last for up to 6 months. Patients will undergo surgical treatment after the preoperative endocrine therapy is completed. Postoperative treatments are then chosen based on the postoperative pathology 
(see "Adjuvant endocrine therapy" for more details). Few clinical studies have compared the values of preoperative endocrine therapy versus preoperative chemotherapy in premenopausal patients. In principle, preoperative endocrine therapy is not recommended for premenopausal patients (except for clinical studies).

\section{Postoperative adjuvant treatment of breast cancer}

\section{Adjuvant treatment for HER-2-positive breast cancer patients without neoadjuvant treatment}

\begin{tabular}{|c|c|c|c|}
\hline Stratification & Level I recommendations & Level II recommendations & Level III recommendations \\
\hline \multirow[t]{2}{*}{ Positive axillary lymph nodes ${ }^{1}$} & $\mathrm{AC} \rightarrow \mathrm{THP}(1 \mathrm{~A})$ & $\mathrm{AC} \rightarrow \mathrm{TH}^{2}(1 \mathrm{~A})$ & \multirow[t]{2}{*}{$\mathrm{H} \rightarrow$ neratinib $^{3}(2 \mathrm{~A})$} \\
\hline & $\mathrm{TCbHP}(1 \mathrm{~A})$ & $\mathrm{TCbH}^{2}(1 \mathrm{~A})$ & \\
\hline $\begin{array}{l}\text { Negative axillary lymph nodes but with high-risk } \\
\text { factors }^{1}\end{array}$ & $\mathrm{AC} \rightarrow \mathrm{TH}(1 \mathrm{~A})$ & $\mathrm{AC} \rightarrow \mathrm{THP}(2 \mathrm{~A})$ & \multirow[t]{3}{*}{$\mathrm{H} \rightarrow$ neratinib (2B) } \\
\hline 1. Tumor sized $>2 \mathrm{~cm}$ & $\mathrm{TCbH}(1 \mathrm{~A})$ & TCbHP (2A) & \\
\hline 2. Other risk factors (e.g., ER-negative) & & & \\
\hline Negative axillary lymph nodes ${ }^{4}$ and tumor sized $\leq 2 \mathrm{~cm}$ & $\mathrm{TC}+\mathrm{H}(2 \mathrm{~A})^{5}$ & wTH $(2 B)^{6}$ & \multirow[t]{2}{*}{ Chemotherapy $\rightarrow \mathrm{H}^{\top}(2 \mathrm{~B})$} \\
\hline $\begin{array}{l}\text { Positive hormone receptors and no chemotherapy is } \\
\text { required; or, can not tolerate chemotherapy }\end{array}$ & & $\mathrm{H}+$ endocrine therapy $^{8}(2 \mathrm{~A})$ & \\
\hline
\end{tabular}

Note: ${ }^{1}$ Compared with the trastuzumab-containing regimen, the dual-targeted therapy containing pertuzumab and trastuzumab reduced iDFS events; patients with positive lymph nodes benefited the most from the dual blockage. Therefore, for patients at high risk of recurrence, especially for patients with positive axillary lymph nodes, dual-targeted therapy with pertuzumab and trastuzumab is recommended. However, experts disagree that dual-targeted therapy should be considered in all patients who are suitable for single-targeted adjuvant therapy. For patients with negative axillary lymph nodes, other risk factors (e.g., tumor size and ER status) should also be considered before an optimal treatment protocol is made.

${ }^{2}$ After a 10 -year long-term follow-up, study showed $\mathrm{TCbH}$ and $\mathrm{AC} \rightarrow \mathrm{TH}$ had similar long-term efficacies; however, the incidence of cardiac insufficiency was lower in the TCbH group. Therefore, TCbH may be selected for patients with higher requirement on cardiac safety.

${ }^{3}$ According to the ExteNET study (8), for patients who have completed trastuzumab therapy and are at risk of recurrence, sequential neratinib may be considered. For patients who have not completed trastuzumab, dual-targeted therapy with trastuzumab and pertuzumab should be considered first.

${ }^{4}$ Indications for adjuvant therapy with trastuzumab for HER-2-positive breast cancer patients with negative axillary lymph nodes: (I) patients with stage T1c or higher breast cancer should receive adjuvant trastuzumab therapy; (II) adjuvant trastuzumab therapy is recommended for patients with stage T1b breast cancer; (III) adjuvant trastuzumab therapy can be considered for patients with stage T1a breast cancer, especially those with high-risk factors such as hormone receptor negative, poor grade, and high Ki-67. At present, there is no clear evidence that patients with HER-2-positive micro-invasive breast cancer can benefit from adjuvant targeted therapy, and thus these patients are not included in the potential candidates for neoadjuvant therapy. However, when the pathological diagnosis is micro-infiltration, the pathological diagnostic criteria should be strictly followed to avoid the misdiagnosis of stage T1a breast cancer as micro-infiltration. A second pathological examination may be performed if necessary.

${ }^{5}$ Subjects in the vast majority of the previous clinical studies were patients with $\mathrm{T} 2$ or higher breast cancer; however, patients with HER-2-positive and lymph node-negative small tumors are still at high risk of recurrence (compared with those with HER-2-negative small tumors). Studies have shown that the 5-year risk of recurrence/metastasis in HER-2-positive $\mathrm{T}_{\text {lab }} \mathrm{N}_{0} \mathrm{M}_{0}$ patients is more than 5 times that of HER-2-negative patients (9).

${ }^{6}$ The APT study (10) suggested that for HER-2-positive patients with primary lesions $\leq 1 \mathrm{~cm}$ and negative lymph nodes, the less toxic wTH regimen may be considered.

${ }^{7}$ It is recommended to use adjuvant trastuzumab therapy as early as possible after surgery. If trastuzumab is not timely 
combined with adjuvant chemotherapy, trastuzumab therapy should be started as soon as possible after chemotherapy. Even if adjuvant chemotherapy has already completed and the patient has not suffered from recurrence/metastasis within 5 years after the adjuvant chemotherapy, use of trastuzumab can still be considered. At present, 1-year trastuzumab remains the standard adjuvant treatment. A large number of clinical studies have verified that a short (6 months or 9 weeks) trastuzumab therapy cannot achieve the same efficacy as 1-year standard therapy. Experts believe that it is currently impossible to identify populations that can best benefit from short-course adjuvant trastuzumab therapy and thus this strategy is not recommended. ${ }^{8}$ As trastuzumab may increase cardiotoxicity, concurrent use with anthracycline is not recommended; however, trastuzumab can be used concurrently with adjuvant radiotherapy and adjuvant endocrine therapy. For hormone receptor-positive patients, endocrine therapy combined targeted therapy can be considered in low-risk patients who do not need chemotherapy or in patients who need chemotherapy but cannot tolerate it.

\section{Adjuvant chemotherapy for HER-2-negative breast cancer $^{1-3}$}

\begin{tabular}{|c|c|c|c|}
\hline Stratification & Level I recommendations & Level II recommendations & Level III recommendations \\
\hline Patients at high risk of recurrence: & $A C \rightarrow T^{5,6}(1 A)$ & $\operatorname{TAC}^{8}$ (IB) & FACX6 \\
\hline 1. $\geq 4$ positive axillary lymph nodes & $\mathrm{ddAC} \rightarrow \mathrm{ddT}^{7}(1 \mathrm{~A})$ & $\mathrm{FEC} \rightarrow \mathrm{T}(\mathrm{IB})$ & (2B) \\
\hline \multicolumn{4}{|c|}{ 2. 1-3 positive lymph nodes along with other risk } \\
\hline \multicolumn{4}{|l|}{ 3. Triple-negative breast cancer ${ }^{4}$} \\
\hline Patients at a lower risk of recurrence: & $A C^{11}(1 A)$ & $\mathrm{AC} \rightarrow \mathrm{T}(2 \mathrm{~A})$ & \\
\hline 1. 1-3 positive lymph nodes (Luminal A) $)^{9}$ & $\mathrm{TC}^{12}(1 \mathrm{~A})$ & & \\
\hline \multicolumn{4}{|l|}{ 2. High Ki-67 expression $(\geq 30 \%)^{10}$} \\
\hline \multicolumn{4}{|l|}{ 3. $>2 \mathrm{~cm}$} \\
\hline 4. Younger than 35 years & & & \\
\hline
\end{tabular}

Notes: ${ }^{1}$ Adjuvant chemotherapy may be considered if one of the following factors exists: (I) positive axillary lymph nodes; (II) triple-negative breast cancer; (III) HER-2-positive breast cancer (T1b or higher); (IV) tumor sized $>2 \mathrm{~cm}$; and/or (V) histological grade 3. Notably, all the above factors are not the absolute indications for adjuvant chemotherapy. The decision of adjuvant chemotherapy should take into account the clinicopathological characteristics of the tumor, the patient's physiological conditions and underlying diseases, the patient's wishes, and the possible benefits and adverse reactions of chemotherapy.

${ }^{2}$ Relative contraindications of adjuvant chemotherapy: (I) pregnant women must be particularly careful about weighing the risks and benefits of taking chemotherapy medicines; (II) old and frail patients with severe visceral organic diseases.

${ }^{3}$ Principles of adjuvant chemotherapy: (I) the purpose of adjuvant chemotherapy for early breast cancer is to cure the disease; therefore, the chemotherapy should be performed in a standardized manner, including the standard drugs, dosages, treatment cycles, and courses. (II) The selection, dosing, and application of chemotherapy drugs and the management of chemotherapyassociated toxicities are particularly complicated. Factors such as toxicity, individual differences, and comorbidities must be considered. A chemotherapy regimen may be selected according to the patient's risk, tolerance, and personal wishes as well as the background of a clinical trial. Meanwhile, a protocol for preventing nausea/vomiting and bone marrow suppression should be established. (III) Special attention should be paid to the order of administration, infusion time, and dose intensity of chemotherapy drugs during chemotherapy. The drug instructions and the incompatibility of drugs must be strictly followed. (IV) In general, do not reduce the number of cycles in a standard chemotherapy regimen unless there are special circumstances. (V) Generally, adjuvant chemotherapy is not implemented concurrently with endocrine therapy or radiotherapy. Endocrine therapy may be started after the chemotherapy cycle is over. Radiotherapy and endocrine therapy can be carried out either sequentially or concurrently.

${ }^{4}$ For some patients with triple-negative breast cancer, if there are known BRCA mutations, platinum (e.g., cisplatin and carboplatin) may be added on the basis of anthracyclines and taxanes. Most experts believe that platinum should be considered 
in neoadjuvant therapy for TNBC patients.

${ }^{5}$ In the HR-negative subgroup of CALGB9344 study, the sequential paclitaxel group achieved prolonged DFS. Therefore, $\mathrm{AC} \rightarrow \mathrm{T}$ chemotherapy is currently recommended for patients at relatively high risk of recurrence.

${ }^{6,7}$ Regarding the selection of taxanes in the $\mathrm{AC} \rightarrow \mathrm{T}$ regimen, three-week docetaxel regimen or the weekly paclitaxel regimen was more effective than the three-week paclitaxel regimen. Dose-dense AC $\rightarrow \mathrm{T}$ regimen can be used for partially tolerable high-risk breast cancer patients.

${ }^{8} \mathrm{AC} \rightarrow \mathrm{T}$ was not significantly different from TAC adjuvant chemotherapy in terms of DFS and OS, but the hematological toxicities in the sequential group was significantly lower than those in the combination group.

${ }^{9}$ For patients with Luminal A or B breast cancer, their chemotherapy regimens depend on the response to chemotherapy and the risk of disease recurrence. Most experts believe that Luminal A breast cancer responds poorly to chemotherapy. If there are indications for chemotherapy (e.g., 1-3 positive lymph nodes), AC or TC may be recommended; for high-risk patients with 4 or more positive lymph nodes, $\mathrm{AC} \rightarrow \mathrm{T}$ is recommended.

${ }^{10} \mathrm{Ki}-67$ expression is an important marker for chemotherapy selection. For low-risk patients (e.g., HR-positive and T1N0), adjuvant chemotherapy is recommended if $\mathrm{Ki}-67>30 \%$; if $\mathrm{Ki}-67$ is $<15 \%$, adjuvant chemotherapy is not currently recommended because its benefit is still not clear; if $\mathrm{Ki}-67$ is between $15 \%$ and $30 \%$, multi-gene testing can be considered, and a decision of adjuvant chemotherapy may be made after communicating effectively with patients about their willingness, tolerance to chemotherapy, and possible benefits and risks of chemotherapy.

${ }^{11}$ The NSABPB-15 study showed that 4 cycles of AC was equivalent to the CMF, with similar toxicities; furthermore, the $\mathrm{AC}$ protocol had shorter treatment course. Thus, AC can be used as a basic regimen for some intermediate- and low-risk patients who require adjuvant chemotherapy. The US9735 study showed that the TC brought improvements in DFS and overall survival (OS). Therefore, for some intermediate- and low-risk patients who require adjuvant chemotherapy, especially when there is a hidden risk of anthracycline cardiotoxicity, adjuvant chemotherapy with TC is also preferred.

${ }^{12}$ There are limited international evidences on the use of pirarubicin; based on the availability of pirarubicin and the clinical experience in China, pirarubicin can be used as an anthracycline, with a commonly recommended dose of $50 \mathrm{mg} / \mathrm{m}^{2}$.

\section{Adjuvant endocrine therapy for hormone receptor-positive breast cancer}

(I) Adjuvant endocrine therapy is particularly important for hormone receptor (ER/PR)-positive breast cancer patients. For the criteria of hormone receptor positive, please refer to the "Molecular typing".

(II) The biological behavior of weakly ER-positive breast cancer (with a positive rate of 1-9\%) is similar to that of ERnegative breast cancer. Therefore, adjuvant chemotherapy should not be abandoned in these patients. After adjuvant chemotherapy is completed, adjuvant endocrine therapy can be considered as appropriate. However, for premenopausal patients with ER-positive 1-9\% breast cancer, ovarian function suppression combined with oral endocrine drugs is not recommended.

(III) Concurrent adjuvant chemo-endocrine therapy is not recommended.

(IV) Ovarian function is a key indicator in the selection of adjuvant endocrine therapy. No matter whether the patient is receiving chemotherapy or not, the patient's menstrual status must be inquired before the commencement of systemic treatment, which helps to determine the patient's ovarian function status and informs the development of full-course adjuvant treatment regimen(s).

Definition of menopause: Menopause can be divided into natural menopause and artificial menopause. Generally, it refers to the permanent cessation of menstruation, which indicates that the estrogen synthesized by the ovaries continuously decreases. Women will be considered menopausal if they meet any of the following criteria:

(i) Bilateral oophorectomy;

(ii) Age $\geq 60$ years;

(iii) Age $<60$ years and with amenorrhea for 12 or more months in the absence of chemotherapy, tamoxifen, toremifene, or ovarian suppression and follicle stimulating hormone (FSH) and estradiol in the postmenopausal range;

(iv) If taking tamoxifen or toremifene and age $<60$ years old, FSH and plasma estradiol levels should be in post-menopausal range in two consecutive measurements. 
Adjuvant endocrine therapy strategies for postmenopausal breast cancer patients Initial therapy

\begin{tabular}{|c|c|c|c|}
\hline Treatment phase & Level I recommendations & Level II recommendations & Level III recommendations \\
\hline \multirow[t]{2}{*}{ Initial therapy } & 1. Al for 5 -year treatment ${ }^{1}(1 A)$ & $2-3$ years of TAM & 5 years of TAM $(2 B)$ \\
\hline & $\begin{array}{l}\text { 2. Patients who initially receive tamoxifen as adjuvant } \\
\text { therapy can switch to } \mathrm{Al} \text { for } 5 \text {-year treatment }{ }^{2,3}(1 \mathrm{~A})\end{array}$ & $\begin{array}{l}\text { Followed by } \mathrm{Al} \text { for } 2-3 \text { years } \\
(2 \mathrm{~A})\end{array}$ & \\
\hline \multicolumn{4}{|l|}{ Extended therapy } \\
\hline \multicolumn{2}{|l|}{ Treatment phase } & Level I recommendations & Level II recommendations \\
\hline \multicolumn{4}{|c|}{ (1) Positive lymph nodes; } \\
\hline \multicolumn{4}{|l|}{ (2) G3; } \\
\hline
\end{tabular}

Notes: ${ }^{1}$ The 10-year follow-up data from the ATAC study (11) confirmed the role of AI as a standard regimen for adjuvant treatment of early breast cancer in postmenopausal patients. Therefore, the AI 5-year therapy is recommended as the initial adjuvant endocrine therapy for postmenopausal patients. For patients having medical contraindications to AI use, TAM may be considered for initial adjuvant endocrine therapy.

${ }^{2}$ The MA17 study confirmed the feasibility and effectiveness of switching to AI for 2-5 years after initial adjuvant treatment with TAM.

${ }^{3}$ According to the results of the BIG1-98 study (12), the switching strategy was more suitable for patients who could not tolerate the initial regimen. During the treatment with AI or TAM, patients should be instructed to properly deal with adverse drug reactions. Switch between AI and TAM if either drug can not be tolerated. For instance, if AI can not be tolerated during the initial treatment, TAM can be used instead.

${ }^{4}$ The initial adjuvant endocrine therapy with AI can be discontinued after 5 years of use in postmenopausal low-risk breast cancer patients. "Low risk" is defined as patients who meet all of the following conditions: postoperative pT $\leq 2 \mathrm{~cm}$; G1; negative lymph nodes; without peritumoral vascular invasion; ER- and/or PR-positive; and HER-2-negative.

${ }^{5}$ After initial 5 years of adjuvant AI therapy, if the patient tolerates it well and meets any of the following conditions, extending the endocrine therapy may be considered: (I) positive lymph nodes; (II) G3; (III) with other risk factors (e.g., Ki67 >30\%) that require adjuvant chemotherapy;

${ }^{6}$ As found in the MA17R study, patients received 5 additional years of AI after initial treatment with TAM and AI further reduced the risk of recurrence. In the NSABP B-42 trial, it was found the extended AI group had significantly lower risk of breast cancer recurrence. These evidences supported the clinical application of extended AI therapy. However, there is still controversy about whether a well-tolerated AI therapy should be further extended.

${ }^{7}$ No randomized controlled trial has explored the role of 5 years of AI followed by 5 years of TAM or AI in postmenopausal patients. However, since some previous studies have demonstrated that switching to AI for another 5 years after 5 years of TAM treatment can be beneficial, switching to 5-year TAM treatment may be feasible in patients who require extended therapy but can not tolerate AI treatment. 


\section{Adjuvant endocrine therapy strategies for premenopausal breast cancer patients}

Initial treatment

\begin{tabular}{|c|c|c|c|}
\hline Stratification & Level I recommendations & Level II recommendations & Level III recommendations \\
\hline $\begin{array}{l}\text { Patients at low risk of recurrence } \\
\text { (must meet all of the following conditions): }\end{array}$ & \multirow[t]{4}{*}{5 years of $\operatorname{TAM}^{1}(1 \mathrm{~A})$} & & \\
\hline (2) G1; & & & \\
\hline (3) $\mathrm{T} \leq 2 \mathrm{~cm}$; & & & \\
\hline (4) Low Ki-67 index; & & & \\
\hline Meeting any of the following conditions: & \multirow[t]{4}{*}{$\begin{array}{l}\text { OFS + TAM 5-year } \\
\text { treatment }^{2-5}(1 \mathrm{~A})\end{array}$} & \multirow[t]{4}{*}{$\begin{array}{l}\text { OFS + AI 5-year treatment }{ }^{4-7} \\
(2 A)\end{array}$} & \multirow[t]{4}{*}{ TAM (2B) } \\
\hline (1) G2 or G3; & & & \\
\hline (2) 1-3 positive lymph nodes; & & & \\
\hline (3) $\mathrm{T}>2 \mathrm{~cm}$; & & & \\
\hline Patients with 4 or more positive lymph nodes & $\begin{array}{l}\text { OFS + Al 5-year treatment }{ }^{4-7} \\
(1 \mathrm{~A})\end{array}$ & $\begin{array}{l}\text { OFS + TAM 5-year treatment }{ }^{1} \\
(2 A)\end{array}$ & TAM (2B) \\
\hline
\end{tabular}

\section{Extended therapy ${ }^{8}$}

\begin{tabular}{|c|c|c|}
\hline Stratification & Level I recommendations & Level II recommendations \\
\hline \multirow[t]{2}{*}{$\begin{array}{l}\text { Patients who have completed the initial TAM } \\
5 \text {-year treatment and require extended therapy }{ }^{9}\end{array}$} & $\begin{array}{l}\text { 1. Extended adjuvant TAM for up to } 10 \text { years in } \\
\text { premenopausal patients }(1 \mathrm{~A})\end{array}$ & \\
\hline & $\begin{array}{l}\text { 2. Al can be used sequentially for } 5 \text { years in } \\
\text { patients whose menopause is confirmed }(1 A)\end{array}$ & \\
\hline $\begin{array}{l}\text { Patients who have completed the initial } 5 \text { years } \\
\text { of OFS + TAM, and the therapy is well tolerated }\end{array}$ & $\begin{array}{l}\text { Sequential Al treatment for menopausal patients } \\
(2 \mathrm{~A})\end{array}$ & $\begin{array}{l}\text { Premenopausal patients treated } \\
\text { with TAM for } 5 \text { years }(2 \mathrm{~B})\end{array}$ \\
\hline $\begin{array}{l}\text { Patients who have completed the initial } 5 \text { years } \\
\text { of OFS }+\mathrm{Al} \text {, and the therapy is well tolerated }{ }^{10}\end{array}$ & Al treatment for menopausal patients $(2 \mathrm{~A})$ & $\begin{array}{l}\text { Premenopausal patients treated } \\
\text { with TAM for } 5 \text { years }(2 \mathrm{~B}) \text { or OFS + } \\
\text { Al for } 5 \text { years }(2 \mathrm{~B})\end{array}$ \\
\hline
\end{tabular}

Notes: ${ }^{1}$ The SOFT study (13) compared the efficacies between OFS + TAM and TAM alone for 5 years. Most of the patients in the pre-set postoperative non-adjuvant chemotherapy subgroup were lymph node negative, Gl, and $\mathrm{T}<2 \mathrm{~cm}$, and subgroup analysis showed that there was limited benefit from OFS + TAM therapy. Therefore, it is recommended that the basic strategy of postoperative adjuvant endocrine therapy for these patients is TAM 5-year treatment.

${ }^{2}$ Strategies used for ovarian suppression include drug-induced ovarian function suppression (e.g., GnRH agonists (GnRHa) such as goserelin and leuprolide) and surgery. Ovarian radiotherapy has been available but it is not routinely recommended. Be wary of the possibility of incomplete drug-induced ovarian function suppression; however, it is not recommended to routinely monitor hormone levels during the use of GnRHa (14).

${ }^{3}$ The pre-set chemotherapy subgroup in the SOFT study showed that patients with positive lymph nodes, higher tumor grade $(2-3)$ and large size $(>2 \mathrm{~cm})$ were more likely to benefit from the OFS combinations.

${ }^{4}$ Notably, the impact of OFS combined with oral endocrine drugs on the survival benefit of breast cancer patients remains unclear and warrants long-term follow-up.

${ }^{5}$ The possible adverse reactions and tolerability of OFS + TAM or OFS + AI must be adequately evaluated, and the adverse reactions of these two combinations must be weighed before making a treatment decision.

${ }^{6}$ The TEXT\&SOFT joint analysis (15) confirmed the benefit of OFS + AI 5-year treatment for patients receiving chemotherapy. Further comprehensive quantitative analysis (16) revealed that factors associated with the absolute benefit of 
OFS $+\mathrm{AI}$ included age $<35$ years, $\geq 4$ positive lymph nodes, and histological gradel 3 . Thus, patients with the above factors are more likely to benefit from OFS plus AI.

${ }^{7}$ No relevant research has been carried out in patients who are pre-menopausal at the time of initial treatment but will enter menopause within 2 to 3 years. The expert group proposes the following options for this population: (I) for patients with 4 or more positive lymph nodes or had a histological grade 3 tumor, ovariectomy followed by AI is recommended. (II) For G2 patients with 1-3 positive lymph nodes, TAM can be selected for the initial adjuvant treatment, and AI can be used instead for 5 additional years after menopause.

${ }^{8}$ After 5 years of initial therapy, if the patient tolerates it well and meets any of the following conditions, extending the endocrine therapy may be considered: (I) positive lymph nodes; (II) G3; (III) younger than 35 years at diagnosis; (IV) high Ki67 expression; (V) pT2 and higher.

${ }^{9}$ Several randomized controlled trials demonstrated that TAM 10-year treatment was superior to TAM 5-year treatment in breast cancer recurrence rate. For patients who have already selected TAM for the initial treatment and have not yet entered menopause after 5 years, it is recommended to extend the TAM treatment for another 5 years (up to 10 years) if such extension is required.

${ }^{10}$ The risk of long-term recurrence persists even after 5 years of OFS combined with oral endocrine drugs. No study has explored the results of extended endocrine therapy in these patients, and no randomized controlled trial has compared the efficacies of the extended endocrine therapy following 5 years of OFS + endocrine drugs versus TAM 10-year treatment. Nevertheless, based on the evidence for the benefit of extended endocrine therapy, extended endocrine therapy can be recommended for patients who can tolerate it.

\section{Adjuvant radiotherapy after breast cancer surgery}

After breast-conserving surgery ${ }^{1}$

\begin{tabular}{|c|c|c|}
\hline Stratification & Level I recommendations & Level II recommendations \\
\hline Negative axillary lymph nodes & $\begin{array}{l}\text { 2. Accelerated partial breast } \\
\text { irradiation }(\mathrm{APBI})(2 \mathrm{~A})^{3}\end{array}$ & $\begin{array}{l}\text { 2. Regional lymph node radiation is recommended for } \\
\text { high-risk patients }(2 \mathrm{~B})^{7}\end{array}$ \\
\hline $\begin{array}{l}\text { (1) Positive axillary lymph nodes, after } \\
\text { axillary lymph node dissection }\end{array}$ & $\begin{array}{l}\text { WBI + tumor bed boost + regional } \\
\text { lymph node radiotherapy }{ }^{7}(1 \mathrm{~B})\end{array}$ & $\begin{array}{l}\text { For low-risk stage II patients, WBI + tumor bed boost } \\
\text { can be considered }(2 \mathrm{~B})^{7}\end{array}$ \\
\hline $\begin{array}{l}\geq 3 \text { positive sentinel lymph nodes, } \\
\text { without axillary lymph node dissection }\end{array}$ & & $\begin{array}{l}\text { WBI + tumor bed boost + regional lymph node } \\
\text { radiotherapy (including axilla) (2B) }\end{array}$ \\
\hline
\end{tabular}


After smastectomy ${ }^{1}$

\begin{tabular}{|c|c|c|}
\hline Stratification & Level I recommendations & Level II recommendations \\
\hline (2) Positive axillary lymph nodes & & \\
\hline
\end{tabular}

Positive sentinel lymph nodes, without axillary lymph node dissection
Chest wall radiotherapy + regional lymph node radiotherapy (including axilla) ${ }^{7}(2 \mathrm{~B})$

Notes: ${ }^{1}$ This indication of radiotherapy is also applicable to patients after neoadjuvant chemotherapy. Currently, it is recommended that, based on the clinical staging of patients before neoadjuvant therapy and the pathological stage after neoadjuvant chemotherapy, a decision on radiotherapy may be made according to the highest stage of the disease.

${ }^{2}$ Postoperative WBI can reduce the risk of recurrence of DCIS and invasive carcinoma by about $50 \%$ in DCIS patients. Patients may be spared postoperative radiotherapy based on the following considerations: prognostic factors such as patient age, histological grade of tumor, and surgical margins; low-risk DCIS; contraindications to radiotherapy; risks and benefits of radiotherapy; and willingness of patients. However, prospective clinical studies have suggested that patients with "low-risk" DCIS can still benefit from radiotherapy (17).

${ }^{3}$ APBI: refer to the American Society of Therapeutic Radiation Oncology (ASTRO) recommendations (18) for patient selection, or refer to the inclusion criteria in the RAPID and NSABPB-39 studies. IMRT or interstitial implantation is recommended for APBI.

${ }^{4}$ For patients who only require WBI on the breast, the recommended postoperative adjuvant radiotherapy includes: conventional fractions 50 Gy/25 fractions, followed by tumor bed boost 10-16 Gy/5-8 times; or, hyperfraction: 40-42.5 Gy/ 15-16 fractions. While these two schedules are equally effective, the hyperfractionated radiotherapy can save medical resources and lower the medical expenditure. Thus, it is recommended as the preferred schedule. The hyperfractionated radiotherapy is also recommended for WBI with high tangential fields.

${ }^{5}$ Patients who meet the above conditions may be spared postoperative radiotherapy after the risks and benefits of radiotherapy are carefully weighed and the patient's wishes are fully respected.

${ }^{6}$ High tangential fields: it refers to the superior border of the tangential field is placed upwards to cover more low axillary lymph nodes. Typically, the upper border is $2 \mathrm{~cm}$ below the humeral head (19).

${ }^{7}$ Regional lymph node radiation: In patients who have received complete axillary lymph node dissection (basically defined as axillary lymph node dissection at stations 1 and 2, and the number of detected lymph nodes is $\geq 10$ ), the regional lymph node dissection typically include the supraclavicular/subclavian areas on the affected side and the Internal mammary lymph nodes (between the first and the third intercostal spaces). (I) Prophylactic irradiation of internal mammary chain (IMC): most of the published clinical studies and meta-analyses on postoperative adjuvant radiotherapy support the inclusion of internal mammary chain (IMC) in the target areas of regional lymph node irradiation (20). Patients with any of the following conditions may benefit more from prophylactic irradiation of IMC: (i) with $\geq$ positive axillary lymph nodes; (ii) with the primary mass located in the central or medial quadrant, and there is axillary lymph node metastasis; (iii) aged $\leq 35$ years, with axillary lymph node metastasis; (iv) with high possibility of internal mammary lymph node metastasis on preuse imaging, or with pathologically confirmed internal mammary lymph node metastasis. (II) Prophylactic irradiation of axillary lymph node areas: postoperative prophylactic irradiation is not required in axillary lymph node areas where the lymph nodes have been completely dissected. For patients who have positive sentinel lymph nodes but have not undergone complete axillary dissection (in principle, patients who meet the inclusion criteria of the Z0011 study), high tangent irradiation is recommended. Some well-validated nomograms (e.g., http://www3.mdanderson.oig/app/medcalc/ bc_nomogram $2 /$ index.cfin?pagename=nsln) for predicting sentinel lymph node status can also be applied. (III) Axillary lymph node-negative "high-risk" patients: Both the EORTC 22922-10925 study and the MA20 study enrolled different proportions of "high-risk" patients with negative lymph nodes. These high-risk factors included: the primary mass was located in the central or medial quadrant; histologic Level III; negative hormone receptor; diffuse vascular tumor thrombi; and young age. 
However, no specific conclusion was made for such "high-risk" population with negative lymph nodes. Therefore, clinicians may use these risk factors to assist decision-making on whether regional lymph node irradiation is required. (IV) If the outcomes of axillary lymph node dissection do not affect the treatment strategy, axillary radiotherapy can be considered to replace axillary lymph node dissection in patients with positive sentinel lymph nodes. (V) Regional lymph node radiation in stage II patients: most of the published clinical studies and meta-analyses on postoperative adjuvant radiotherapy support that N1 patients can benefit significantly from regional lymph node radiotherapy.

\section{Treatment of advanced breast cancer}

\section{Treatment of HER-2-positive advanced breast cancer $^{1}$}

\begin{tabular}{|c|c|c|c|}
\hline Stratification & Level I recommendations & Level II recommendations & Level III recommendations \\
\hline $\begin{array}{l}\text { 2. Trastuzumab-treated but } \\
\text { meeting the criteria for } \\
\text { reuse }^{3}\end{array}$ & $\begin{array}{l}\text { 2. } \mathrm{TXH} \\
\text { (taxanes + capecitabine }+ \\
\text { trastuzumab) }(1 \mathrm{~A})\end{array}$ & & $\begin{array}{l}\text { 2. Trastuzumab + pertuzumab }+ \\
\text { other chemotherapy drugs }(2 \mathrm{~B})\end{array}$ \\
\hline \multirow[t]{3}{*}{ Trastuzumab treatment failed ${ }^{4}$} & Pyrotinib + capecitabine (1A) & 1. T-DM1 (1A) & 1. Pyrotinib monotherapy ${ }^{7}$ \\
\hline & & 2. Lapatinib + capecitabine (2B) & $\begin{array}{l}\text { 2. TKI combined with other } \\
\text { chemotherapy drugs }(2 \mathrm{~B})\end{array}$ \\
\hline & & & $\begin{array}{l}\text { 3. Trastuzumab in combination with } \\
\text { other chemotherapy drugs (2B) }\end{array}$ \\
\hline
\end{tabular}

Note: ${ }^{1}$ All patients with HER-2-positive recurrent and metastatic breast cancer should be fully informed of the benefits and necessity of receiving HER-2-targeted therapy timely.

${ }^{2}$ Trastuzumab-naive: (I) trastuzumab-based therapy is preferred. A reasonable combination treatment protocol should be selected according to the patient's hormone receptor status and the previous neoadjuvant/adjuvant therapies. (II) Trastuzumab plus taxanes has become the standard first-line therapy for patients with HER-2 + MBC. The CHAT study confirmed that for patients who could tolerate dual-drug chemotherapy, trastuzumab combined with docetaxel plus capecitabine was more effective than trastuzumab combined with docetaxel, especially in patients requiring maintenance treatment (21). (III) The CLEOPATRA study (22) confirmed that the combination of docetaxel with dual-targeted therapy with pertuzumab and trastuzumab was more effective than the combination of docetaxel with trastuzumab. Thus, the combination of docetaxel with pertuzumab and trastuzumab has become the preferred treatment for HER-2 positive patients if therapy with trastuzumab and taxanes has not failed.

${ }^{3}$ Patients who are feasible for reuse with trastuzumab include: (I) showing response to neoadjuvant therapy; (II) having recurrence one year after the end of adjuvant therapy; and (III) drug withdrawal after effective treatment of advanced breast cancer.

${ }^{4}$ Trastuzumab treatment failed: (I) in patients experiencing disease progression after trastuzumab treatment, continuous inhibition of the HER-2 pathway can continue to bring survival benefits. Therefore, after the first-line trastuzumab fails to prevent disease progression, second-line HER-2-targeted therapy is recommended. (II) According to the PHENIX study (23), in patients who failed the treatment with taxanes and trastuzumab, pyrotinib combined with capecitabine was more effective than capecitabine monotherapy in increasing overall response rate (ORR) and progression-free survival (PFS). Therefore, pyrotinib combined with capecitabine was recommended for the treatment of patients who have failed trastuzumab and taxanes. In the same study, patients in the placebo plus capecitabine group received pyrotinib monotherapy sequentially after the disease progressed and still had good benefits. Thus, the expert group believes that pyrotinib monotherapy can also be used as one of the treatment options after trastuzumab failure. The phase II trial of pyrotinib included some trastuzumab- 
naive patients. Therefore, the expert group agrees that pyrotinib combined with capecitabine can also be considered in patients who have not previously failed trastuzumab. (III) The EMILIA study (24) confirmed that, compared with lapatinib combined with capecitabine, T-DM1 had significant PFS and OS benefits. Internationally, T-DM1 is the standard antiHER-2 treatment in the second-line setting. (IV) According to the EGF100151 study and the GBG26 study, if disease progresses despite trastuzumab treatment, the treatment options may include: lapatinib combined with capecitabine (25); or, continue to use trastuzumab but replace the chemotherapy drug.

${ }^{5}$ Target therapy combined with chemotherapy is preferred for HER-2-positive and hormone receptor-positive recurrent/ metastatic breast cancer. For patients who are not suitable for chemotherapy or whose diseases progress slowly, combination with endocrine therapy may be considered on the basis of HER-2-targeted therapy. Research has shown that HER-2-targeted therapy combined with endocrine therapy + CDK4/6 inhibitors has certain efficacy. Therefore, targeted therapy combined with 'endocrine+' therapy may be applied in some patients. In patients who have achieved stable disease after HER-2-targeted therapy combined with chemotherapy, maintenance therapy with HER-2 targeted therapy plus endocrine therapy may be considered after the chemotherapy is stopped.

${ }^{6}$ In patients who are receiving trastuzumab ( \pm pertuzumab) plus chemotherapy, the effective chemotherapy should last at least 6 to 8 cycles, depending on the treatment response and the patient's tolerance to chemotherapy. After chemotherapy is stopped, maintenance treatment with trastuzumab ( \pm pertuzumab) is recommended. If complete response is achieved, the duration of HER-2-targeted therapy should be decided according to factors including drug toxicities and economic burden. In some patients, it may be feasible to suspend anti-HER-2 treatment for 2 to 3 years after complete response; if the disease progresses again, resume the anti-HER-2 treatment that has previously been effective.

${ }^{7}$ Some patients may experience brain metastases during the treatment of HER-2 positive advanced breast cancer. If the extracranial lesions have not progressed, the HER-2-targeted therapy should be continued after effective localized treatments for brain metastases. The initial targeted therapy may be continued or replaced by a TKI.

Chemotherapy of HER-2-negative advanced breast cancer ${ }^{1,2}$

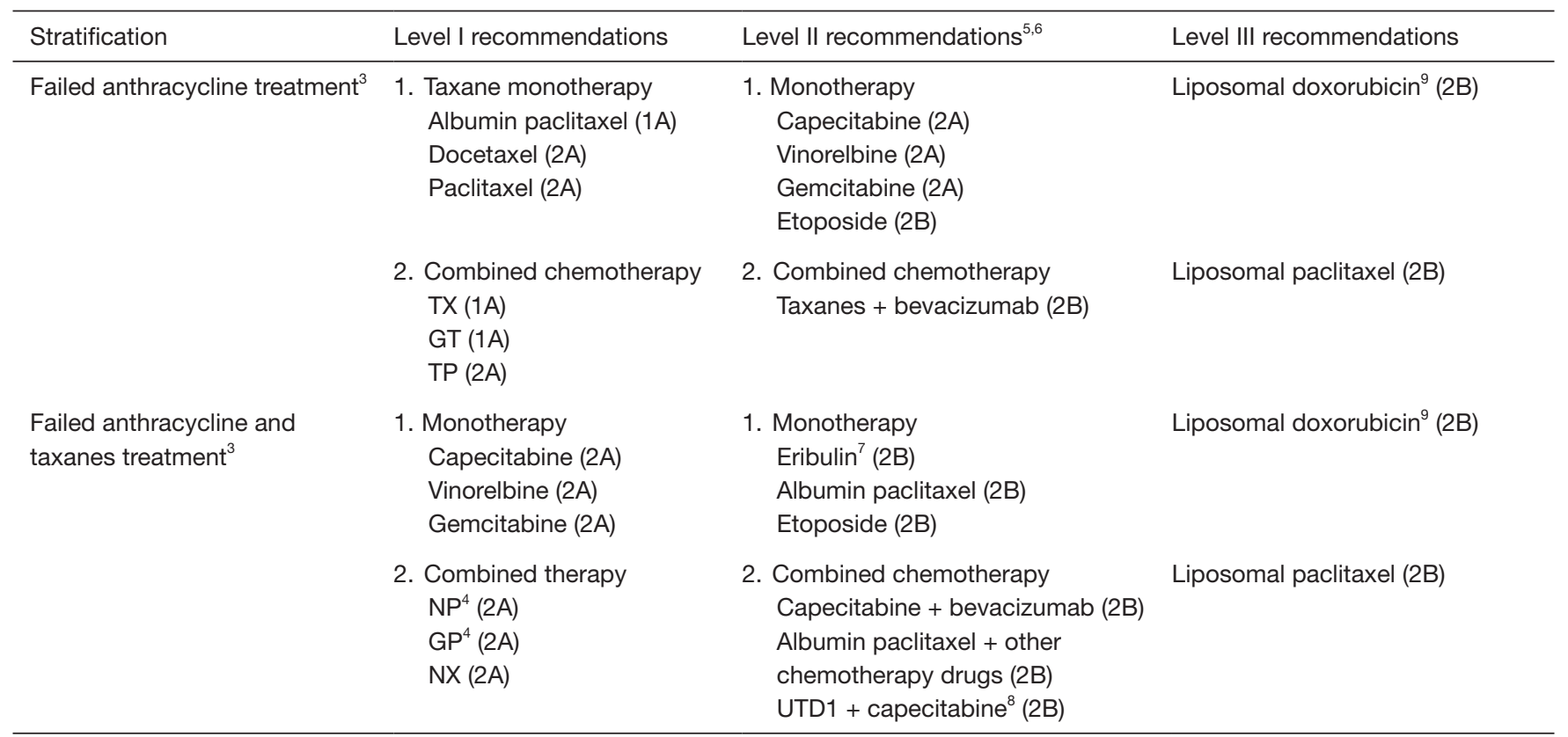


Note: ${ }^{1}$ To the indications of chemotherapy, one of the following factors can be considered as the first choice for chemotherapy. (I) Hormone receptor-negative. (II) Symptomatic visceral metastasis. (III) Hormone receptor-positive but resistant to endocrine therapy.

${ }^{2}$ Principles of chemotherapy for advanced breast cancer. (I) The preferred chemotherapy regimens include single-agent chemotherapy and combination chemotherapy. Compared with the single-agent chemotherapy, the combination chemotherapy usually has a higher objective response rate and longer PFS. However, combination chemotherapy is more toxic and has limited survival benefits. Therefore, combination chemotherapy is only feasible for patients who need to shrink tumors or relieve symptoms within a short period of time. In contrast, single-agent chemotherapy is preferred for patients in whom drug tolerability and quality of life are the top concerns. (II) For recurrent/metastatic breast cancer patients who have failed prior anthracycline-containing preoperative/adjuvant therapy, taxane-based regimens are typically preferred, and both single-agent and combination regimens can be selected for the first-line treatment (26). Other optional drugs may include capecitabine, gemcitabine, vinorelbine, liposomal doxorubicin, and liposomal paclitaxel. (III) There is currently no standard chemotherapy regimen for patients with recurrent/metastatic breast cancer who have failed preoperative/adjuvant treatments with anthracyclines and taxanes. The optional drugs may include capecitabine, vinorelbine, gemcitabine, platinum, eribulin, UTD1, another type of taxane (e.g., albumin paclitaxel), and liposomal doxorubicin. Either single-agent or combination regimens can be considered. (IV) The duration (number of cycles) of each regimen and the feasibility of multi-line chemotherapy should be individualized according to the specific conditions of each patient. For patients who have exhibited responses to combination chemotherapy, a maintenance treatment strategy can be considered after 6 to 8 cycles of combination chemotherapy.

${ }^{3}$ Taxanes (anthracyclines) treatment failure is defined as disease progression during the treatment with taxanes (anthracyclines) for advanced breast cancer (after at least two cycles) or tumor recurrence/metastasis within 12 months after completing the adjuvant therapy. Reuse with taxanes can be considered in the following conditions: (I) the neoadjuvant treatment with taxanes is effective; (II) tumor recurs within one year after adjuvant treatment with taxanes is completed; (III) treatment with taxanes is discontinued after the tumor responds well to the treatment for advanced breast cancer (27).

${ }^{4}$ TNT (28), and other studies have shown that platinum has a high therapeutic effect on triple-negative breast cancer. Platinum-containing regimens can be used as one of the chemotherapy options for triple-negative breast cancer patients, especially in those with BRCA1/2 mutations.

${ }^{5}$ As shown in the Impassion 130 study (29), the combination of PD-L1 antibody with albumin paclitaxel as the first-line treatment of metastatic or unresectable locally advanced triple-negative breast cancer could significantly prolong PFS; in particular, it achieved OS benefit in patients with positive PD-L1 expression. Therefore, the expert group encourages patients with triple-negative advanced breast cancer to actively participate in clinical trials on immune checkpoint inhibitors.

${ }^{6}$ The OlympiAD study (30) showed that, for patients with HER-2-negative advanced breast cancer with BRCA1/2 germline mutations, olaparib could significantly prolong PFS ( $7 v s .4 .2$ months, compared with chemotherapy). Therefore, the expert group generally agrees that patients with BRCA1/2 germline mutations can receive treatment with olaparib or actively participate in relevant clinical studies.

${ }^{7}$ The 304 study (31) showed that for patients with advanced breast cancer for whom anthracyclines and taxanes have failed, eribulin was superior to vinorelbine in prolonging PFS and increasing ORR, with similar incidence of adverse events. Thus, eribulin has become a new option after failure with anthracyclines and taxanes in advanced breast cancer.

${ }^{8}$ The BG01-1312L study (32) showed that for patients with advanced breast cancer for whom anthracyclines and taxanes have failed, UTD1 combined with capecitabine was superior to capecitabine alone in prolonging PFS and OS, which offered a new treatment chance after failure with anthracyclines and taxanes in advanced breast cancer.

${ }^{9}$ During the use of liposomal doxorubicin, it is important to inquire about the patient's previous anthracycline doses, efficacy, treatment course, and possible adverse reactions (especially cardiotoxicity). 
Endocrine therapy for hormone receptor-positive advanced breast cancer ${ }^{1}$

Endocrine therapy for hormone receptor-positive advanced breast cancer in postmenopausal women ${ }^{2}$

\begin{tabular}{|c|c|c|c|}
\hline Stratification & Level I recommendations & Level II recommendations & Level III recommendations \\
\hline \multirow[t]{2}{*}{ Endocrine therapy-naive } & $\mathrm{Al}+\mathrm{CDK}_{4 / 6}$ inhibitors $^{5}(1 \mathrm{~A})$ & 1. $A I^{3}(1 A)$ & TAM (2B) \\
\hline & & 2. Fulvestrant ${ }^{4}(1 \mathrm{~A})$ & \\
\hline \multirow[t]{2}{*}{ Failed TAM treatment } & 1. $A I+C D K 4 / 6$ inhibitors ${ }^{5}(1 A)$ & 1. $A I^{3}(1 A)$ & \\
\hline & 2. $\mathrm{Al}+\mathrm{HDAC}$ inhibitors ${ }^{9}(1 \mathrm{~A})$ & 2. Fulvestrant ${ }^{6}(1 \mathrm{~A})$ & \\
\hline \multirow{3}{*}{$\begin{array}{l}\text { Failed non-steroidal Al } \\
\text { treatment }\end{array}$} & 1. Steroidal $A I+$ HDAC inhibitors ${ }^{9}(1 A)$ & 1. Steroidal AI + CDK4/6 inhibitors (2A) & 1. Steroidal $\mathrm{Al}^{11}(2 \mathrm{~B})$ \\
\hline & 2. Fulvestrant $+C D K 4 / 6$ inhibitors ${ }^{8}(1 \mathrm{~A})$ & 2. Fulvestrant $(2 A)^{7}$ & 2. TAM or toremifene ${ }^{12}(2 \mathrm{~B})$ \\
\hline & & 3. Steroidal $\mathrm{Al}+$ everolimus $(\mathrm{IB})^{10}$ & 3. Progesterone ${ }^{12}(2 \mathrm{~B})$ \\
\hline $\begin{array}{l}\text { Failed steroidal AI } \\
\text { treatment }\end{array}$ & & & 3. Progesterone $e^{12}(2 \mathrm{~B})$ \\
\hline
\end{tabular}

Endocrine therapy for hormone receptor-positive advanced breast cancer in premenopausal women

Effective ovarian function suppression measures (e.g., drugs for ovarian function suppression including goserelin and leuprolide) can be taken. Or, after the ovaries are surgically removed, the subsequent endocrine therapy may follow the principles of endocrine therapy for postmenopausal patients.

Notes: ${ }^{1}$ Endocrine therapy for advanced breast cancer patients who have the following features: (I) pathology reveals the primary or recurrent/metastatic lesion is ER- and/or PR-positive. (II) The tumor progresses slowly. (III) Previous endocrine therapy is effective (including tumor progresses after the completion of postoperative adjuvant therapy), or the DFS is long (e.g., more than 2 years) during adjuvant therapy, or patients have benefited from treatments for recurrence and metastasis breast cancer. (IV) Research has shown that the disease control rate and PFS of endocrine therapy plus targeted therapy is not inferior to those of chemotherapy. Therefore, experts believe that even for some breast cancer patients with high tumor burden (e.g., with visceral metastasis), endocrine therapy plus targeted therapy (e.g., CDK4/6 inhibitor and HDAC inhibitor) may also be a treatment option.

${ }^{2}$ When selecting a first-line endocrine therapy for recurrent and metastatic breast cancer, the physicians must consider the patient's adjuvant treatment regimen, disease-free interval, and disease burden of recurrence/metastasis.

${ }^{3}$ The results of North American trials and TARGET (33) confirmed that for postmenopausal, hormone receptor-positive patients who have not received endocrine therapy or those who have failed adjuvant endocrine therapy with TAM, the thirdgeneration aromatase inhibitors are recommended as first-line endocrine therapy.

${ }^{4}$ The FALCON study confirmed that fulvestrant can be recommended for first-line endocrine therapy in patients with advanced breast cancer.

${ }^{5}$ The PALOMA-1 study revealed that letrozole combined with a CDK4/6 inhibitor (palbociclib) significantly prolonged PFS. The PALOMA-2 study further confirmed the above conclusion. In the MONALEESA-2 study, letrozole combined with a CDK4/6 inhibitor (ribociclib) significantly prolonged PFS compared to letrozole monotherapy. One CDK4/6 inhibitor (palbociclib) has been licensed in China, and the expert group believes that it can be one of the new endocrine treatments for advanced breast cancer.

${ }^{6}$ China CONFIRM (34) demonstrated that fulvestrant $500 \mathrm{mg}$ was more effective than fulvestrant $250 \mathrm{mg}$ in postmenopausal HR+ breast cancer patients who had been treated with endocrine therapy.

${ }^{7}$ Both Global CONFIRM and China CONFIRM enrolled advanced breast cancer patients with relapse/metastasis after AI 
treatment, and China CONFIRM study confirmed the clinical advantages of fulvestrant $500 \mathrm{mg}$ in AI-treated patients.

${ }^{8}$ In the PALOMA-3 study (35), in patients experiencing disease progression despite prior endocrine therapy (with AI or TAM), including patients progressed during adjuvant endocrine therapy or within 12 months after stopping treatment or those who progressed during endocrine therapy for advanced breast cancer, the combination of CDK4/6 inhibitor (palbociclib) with fulvestrant prolonged PFS and prolonged OS, but the difference was not statistically significant. About $70 \%$ of patients in the MONARCH2 study (36) had previously been treated with AI; it was found that the addition of abemaciclib to fulvestrant resulted in an improvement in PFS.

${ }^{9}$ In the ACE study (37), for postmenopausal HR+/HER-2- advanced breast cancer patients who had previously failed tamoxifen and/or non-steroidal AI treatment, the combination of HDAC inhibitor chidamide with exemestane could significantly prolong PFS; also, it was superior to exemestane in terms of objective remission rate and clinical benefit rate. Chidamide has gotten approval in China for breast cancer indication. The expert group recommends that chidamide combined with $\mathrm{AI}$ can be used in advanced breast cancer patients who have failed on previous endocrine treatment.

${ }^{10}$ The BOLERO-2 study confirmed that, the combined regimen can be a treatment option after the failure of non-steroidal AI. However, special attention should also be paid to the possible adverse reactions including stomatitis (which is most common) and the rare but severe interstitial pneumonia, and the dose should be adjusted as appropriate.

${ }^{11} \mathrm{AIs}$ can be used in patients who experience recurrence more than 12 months after end of adjuvant AI therapy. For patients with recurrence $\leq 12$ months after end of adjuvant treatment or progression after first-line endocrine therapy with AI, use of another AI with different mechanism of action (e.g., use of a steroidal AI after a non-steroidal AI) may be helpful, which, however, has not been supported in large randomized controlled clinical studies. Drugs should be reasonably selected based on the patients general conditions and the availability of drugs in China.

${ }^{12}$ If the disease progresses despite AI treatment, other drugs including progesterone (medroxyprogesterone or megestrol), toremifene, and TAM may also be used in the endocrine therapy for advanced breast cancer. Although there are no relevant evidences from large randomized controlled clinical studies, these drugs may be reasonably selected based on the patients general conditions and the availability of drugs in China.

${ }^{13}$ Definition of endocrine resistance (note: this definition is applied in clinical trial enrollment and is used only for reference in clinical settings). (I) Primary endocrine resistance: primary endocrine resistance is defined as relapse while on the first 2 years of adjuvant endocrine therapy (ET) or progressed within the first 6 months of first-line ET for advanced breast cancer. (II) Secondary (acquired) endocrine resistance: secondary (acquired) endocrine resistance is defined as relapse while on adjuvant endocrine therapy but after the first 2 years of treatment, relapse within 12 months of completing adjuvant endocrine therapy, or progressed after six or more months for metastatic breast cancer.

\section{Bone metastasis of breast cancer}

\section{Diagnosis of bone metastasis}

Basic principles

1. ECT and other examinations should be performed to identify any possible bone metastasis in breast cancer patients who suffer from symptoms such as bone pain or have any of the following conditions: (I) hypercalcemia, elevated alkaline phosphatase, and elevated lactate dehydrogenase; (II) abnormally elevated tumor markers (e.g., CEA and CA153); and/or (III) suspicious bone metastasis on other imaging modalities ${ }^{2}$. However, abnormal tracer uptake on bone scan can not ensure the diagnosis of bone metastases

2. When abnormal tracer uptake is found during ECT, CT or X-ray imaging should be performed on the suspicious site to determine whether there is bone destruction ${ }^{3}$

3. MRI is more sensitive but less specific than CT. MRI has definite advantages over CT in identifying neurovascular compression, vertebral body involvement, and spinal stability. It is an important tool for deciding the appropriateness of surgery and radiotherapy for bone metastases. However, abnormal MRI finding alone is not sufficient to make a diagnosis of bone metastasis, which should be based on other examinations/tests. In addition, MRI should not be used as a response assessment tool for bone metastases

4. Pathological examination of bone biopsy samples can help diagnose breast cancer bone metastases. For clinically suspicious bone metastases, especially in patients with a single bone lesion, bone biopsy ${ }^{4}$ should be performed to confirm the diagnosis 


\section{Anti-bone metastasis therapy}

\section{Basic principles}

1. The application of systemic anti-tumor medications is based on the principle of classified treatment ${ }^{7}$

2. Rational use of bone-modifying agents ${ }^{8}$

Zoledronic acid or ibandronic acid (1A)

Denosumab (1B)

Other bisphosphonates (1B)

3. Surgical treatment ${ }^{9}$

4. Local radiotherapy ${ }^{10}$

\section{Recommendation on bone-modifying agents}

\begin{tabular}{lll}
\hline Level I recommendations & Level II recommendations & Level III recommendations \\
\hline Zoledronic acid (1A) & Denosumab (1B) & Clodronate disodium (2B) \\
lbandronic acid (2A) & Loading-dose ibandronic acid (2A) & \\
& Pamidronate disodium (1B) & \\
\hline
\end{tabular}

Notes: ${ }^{1}$ The definition of skeletal-related events (SREs) in clinical studies: SREs include intensified bone pain or new bone pain, pathological fractures (vertebral fractures or non-vertebral fractures), vertebral compression or deformation, spinal cord compression, symptoms following bone radiotherapy (for bone pain or for the prevention/treatment of pathological fractures or spinal cord compression), and hypercalcemia. SREs such as bone pain and bone injury are common symptoms of breast cancer bone metastasis and can seriously undermine quality of life. SREs are the main factors affecting the patient's ability to move independently and the quality of life. Among them, spinal cord compression is a cancer-related emergency requiring multidisciplinary consultations (including the inputs of orthopedic experts). Corticosteroids and other treatments for dehydration should be applied to promptly relieve the compression and reduce the limb dysfunction and even paraplegia caused by spinal cord compression.

${ }^{2}$ Emission computed tomography (ECT) of bones is the most commonly used method for screening bone metastasis. Bone ECT is recommended for the routine screening of breast cancer patients with suspicious bone metastasis symptoms including bone pain, pathologic fracture, increased alkaline phosphatase level, and hypercalcemia. It can also be used for routine examinations in patients with locally advanced breast cancer (above T3N1M0) and/or recurrent/metastatic breast cancer. When bone ECT is used for evaluating treatment response, an increasing number of sites with increased tracer uptake does not necessarily mean disease progression. Bone-window CT scan should be performed. If the original osteolytic lesions have been transformed into bone calcification and the new sites with increased tracer uptake also have the appearance of bone calcification, it can be evaluated as "PR/CR". If the new sites with increased tracer uptake exhibit the appearance of osteolytic lesions, it can be evaluated as "PD".

${ }^{3}$ Bone CT and X-ray examinations are the main imaging methods for clinical diagnosis of bone metastases. In patients with abnormal ECT findings, MRI, CT (bone window), and x-ray should be performed in sites with suspicious bone metastatic lesions, so as to confirm the possible bone metastasis and learn the severity of bone destruction.

${ }^{4}$ Bone biopsy is an invasive examination. When the imaging findings are inconsistent with the clinical findings, it is recommended to perform bone biopsy on the suspected area to determine whether the affected bone is the result of a bone metastasis. Bone biopsy should also be considered in patients with a single suspicious lesion.

${ }^{5}$ FDG-PET has similar sensitivity with bone ECT. However, the expert panel believes that the value of PET/CT in diagnosing bone metastasis still requires further investigations. Currently it is not routinely recommended in clinical settings. ${ }^{6}$ Bone metastases of breast cancer are frequently seen as multiple osteolytic lesions. In some patients, the repaired osteolytic lesions after treatment may be radiologically seen as excessive calcification and thus misdiagnosed as osteogenic changes. In these patients, the X-ray or CT film at the first presentation should be reviewed to identify the presence of any osteolytic lesion. A diagnosis of bone metastases cannot be made if there is only abnormal bone ECT finding and/or abnormally 
elevated alkaline phosphatase or lactate dehydrogenase but without abnormal findings on MRI, CT, or X-ray. Reexaminations with bone ECT or bone CT within 3 months is recommended. If there are more site with increased tracer uptake, further examinations should be performed to confirm the diagnosis. A multidisciplinary analysis is required based on clinical symptoms, tumor markers, and imaging changes. Avoid a subjective judgment based on symptoms only; also, avoid make a decision based on the imaging findings only without inquiring about pain symptoms and change in quality of life.

${ }^{7}$ The treatment of breast cancer bone metastasis should be based on systemic therapies including chemotherapy, endocrine therapy, and molecularly targeted therapy. Factors affecting the choice of systemic treatment include the hormone receptor status and HER2 status of a breast cancer, patient's age and menstrual status, and whether the disease progresses slowly. Breast cancer bone metastasis generally is not directly life-threatening, and breast cancer bone metastasis patients without visceral metastasis usually have relatively long survival; thus, endocrine therapy is preferred in patients with hormone receptorpositive tumors that progress relatively slowly and without primary endocrine resistance. For patients with ER- and PRnegative tumors, short postoperative disease-free interval, rapid disease progression, or HR-positive tumors and with primary endocrine resistance, single-agent chemotherapy is preferred in patients with a single bone metastasis or asymptomatic visceral metastasis, whereas combination chemotherapy should only be considered for patients with bone metastases requiring rapid symptom control or those with symptomatic visceral metastases. For patients with HER-2-positive bone metastases, the treatment principle is the same as that for patients with metastases to other sites - combination with an HER-2-targeted therapy is preferred.

${ }^{8}$ Administration advice:

(I) Serum electrolyte levels, particularly serum creatinine, calcium, phosphate, and magnesium levels, should be measured before the use of bisphosphonates.

(II) Calcium and vitamin D should be supplemented on a daily basis after long-term use of bisphosphonate combinations (calcium: 1,200-1,500 mg/d; Vitamin D: 3,400-800 IU).

(III) No dose adjustment is needed in patients with mild or moderate renal insufficiency (creatinine clearance larger than $30 \mathrm{~mL} / \mathrm{min}$ ). However, for patients with severe renal insufficiency (creatinine clearance less than or equal to $30 \mathrm{~mL} / \mathrm{min}$ ), the dose should be adjusted or the duration of infusion should be prolonged in accordance with different product specifications. Patients with creatinine clearance less than $30 \mathrm{~mL} / \mathrm{min}$ or on dialysis should be closely monitored during treatment with desulumab to avoid the occurrence of hypocalcemia.

(IV) It has been reported that bisphosphonate-associated osteonecrosis of the jaw (BONJ) is a morbid bone disease linked to long-term bisphosphonate use. Therefore, oral examinations and appropriate prophylactic treatments should be performed before using bisphosphonates. During medication, attention should be paid to oral hygiene, and oral surgeries such as tooth extraction should be avoided as possible. If there are persisting areas of exposed bone in the maxillofacial region that occur without a known reason during medication or after a surgery, consult a specialist as soon as possible.

${ }^{9}$ Indications for discontinuation of therapy:

(I) Adverse reactions are detected during drug administration and they are clearly related to the use of bone-modifying agents;

(II) The tumor progresses during the treatment as it spreads to other organs and can be life-threatening;

(III) The clinicians believe it is necessary to stop the medications;

(IV) Relief of bone pain after treatment is not an indication for discontinuation of therapy.

${ }^{10}$ Surgical treatment of bone metastasis

The purposes of surgical treatment are to improve the patients' quality of life by resolving nerve compression, alleviating pain, and restoring limb functions. Patients with bone metastasis must be closely followed up. A proper decision-making on the surgery on the long bones with potentially pathological fractures is critically important to improve the patients' quality of life if an effective surgical treatment is performed before fracture and/or before spinal cord compression.

Surgical procedures for breast cancer bone metastases include simple internal fixation, debridement plus internal fixation, lesion resection plus artificial joint replacement, decompression after spinal cord compression, and reconstruction for increasing spinal stability. Fixation can be selectively applied for pathological fracture or spinal cord compression, especially in breast cancer bone metastasis patients with an expected survival time of $>3$ weeks. Prophylactic fixation can be selectively applied in breast 
cancer bone metastasis patients with one or more of the following conditions: femoral metastasis sized $>2.5 \mathrm{~cm}$; metastasis to the neck of femur bone; bone cortical destruction $>5 \%$; and/or with an expected survival time of $>3$ months.

${ }^{11}$ Radiotherapy for bone metastasis:

The main role of radiotherapy in patients with breast cancer bone metastasis is to relieve bone pain and reduce the risk of pathological fractures.

Radiotherapy includes external-beam irradiation and radionuclide therapy. External-beam irradiation is a common effective method during the palliative therapy for bone metastases. The main indications of external-beam irradiation include: symptomatic bone metastases, for which the treatment is to alleviate pain and restore functions; and, selectively used for the prophylactic radiotherapy of metastasis to weight-bearing bones (e.g., spine and femur). The doses of externalbeam irradiation include $30 \mathrm{~Gy} / 10$ fractions, $20 \mathrm{~Gy} / 5$ fractions, and $8 \mathrm{~Gy} /$ fraction. Although these doses have comparable effectiveness in stopping acute analgesia, long-course radiotherapy can achieve longer pain control. Clinically, appropriate radiotherapy fractionation should be selected according to the expected survival time and the tolerance dose of normal tissues related to the metastases. Effective external-beam irradiation can achieve objective pain relief in $2 / 3$ of patients and achieve complete pain relief in $1 / 3$ of patients. For patients who are eligible for stereotactic body radiation therapy (SBRT), the irradiation dose can be increased and the normal tissues can be better protected.

Radionuclide therapy is useful in easing diffuse pain due to bone metastasis; however, in some patients, radionuclide therapy may result in high incidence of bone marrow suppression, which can only be restored slowly (about 12 weeks) and thus affect the implementation of chemotherapy. Therefore, the clinical use of radionuclide therapy should only be performed in carefully selected cases at the right time.

\section{Brain metastasis of breast cancer}

\section{Clinical features of breast cancer brain metastasis}

Brain metastasis ${ }^{1}$ includes parenchymal and meningeal metastases.

The clinical manifestations of brain parenchymal metastasis mainly include increased intracranial pressure and neurological dysfunction. The main symptoms and signs of increased intracranial pressure are headache, vomiting, and optic disc edema, which may be accompanied by increased blood pressure, visual disturbances, disturbances of consciousness, and incontinence. Due to the different locations of brain metastases, different symptoms and signs such as mental symptoms, epileptic seizures, local sensory/movement disorders of limbs, aphasia, and visual field damage may occur.

Meningeal metastasis is often associated with meningeal irritation, which is manifested as headache, vomiting, stiff neck, cognitive impairment, confusion, and epileptic seizures. It may be accompanied by cranial nerve damage and increased intracranial pressure. If the tumor also spreads along spinal membrane, spinal cord and spinal nerve root stimulation can occur, which is manifested as radicular pain and segmental sensory disturbance.

\section{Basic principles of diagnosis of brain metastasis}

Basic principles

1. Contrast-enhanced MRI of the head is more sensitive for small lesions, edema, and meningeal metastases than contrast-enhanced CT and should be used as the preferred imaging method for the diagnosis of brain metastases. Contrast-enhanced CT can be performed in patients with contraindications for cranial MRI

2. PET-CT can reflect the difference in metabolic status between tumors and normal tissues, which is helpful for tumor diagnosis. However, it is not sensitive for small metastases in the brain. Thus, the clinical diagnosis of small metastases should also be based on the findings of contrast-enhanced MRI or contrast-enhanced CT of the head

3. Patients with central nerve metastasis-associated symptoms but without intracranial space-occupying lesions on MRI/CT should undergo lumbar puncture, which not only allows the measurement of cerebrospinal fluid pressure (CSFP) but also enables the routine, biochemical, and cytological examinations of the cerebrospinal fluid (CSF). However, CSF examination in patients with intracranial hypertension may increase the risk of brain herniation 


\section{Anti-brain metastasis therapy}

The aim of treatment is to treat metastatic lesions, improve patient symptoms, improve quality of life, and maximize the survival time of patients. The treatments of breast cancer brain metastasis include surgery, radiotherapy, chemotherapy, and symptomatic/supportive treatment. The general treatment principle is: surgery and/or radiotherapy for brain metastases is preferred after comprehensive whole body assessment, and meanwhile a rational systemic treatment protocol can also be considered. Radiotherapy mainly includes whole brain radiotherapy (WBRT) and stereotactic radiotherapy (SRT). and the aim of treatment is to treat metastatic lesions, improve patient symptoms, improve quality of life, and maximize the survival time of patients.

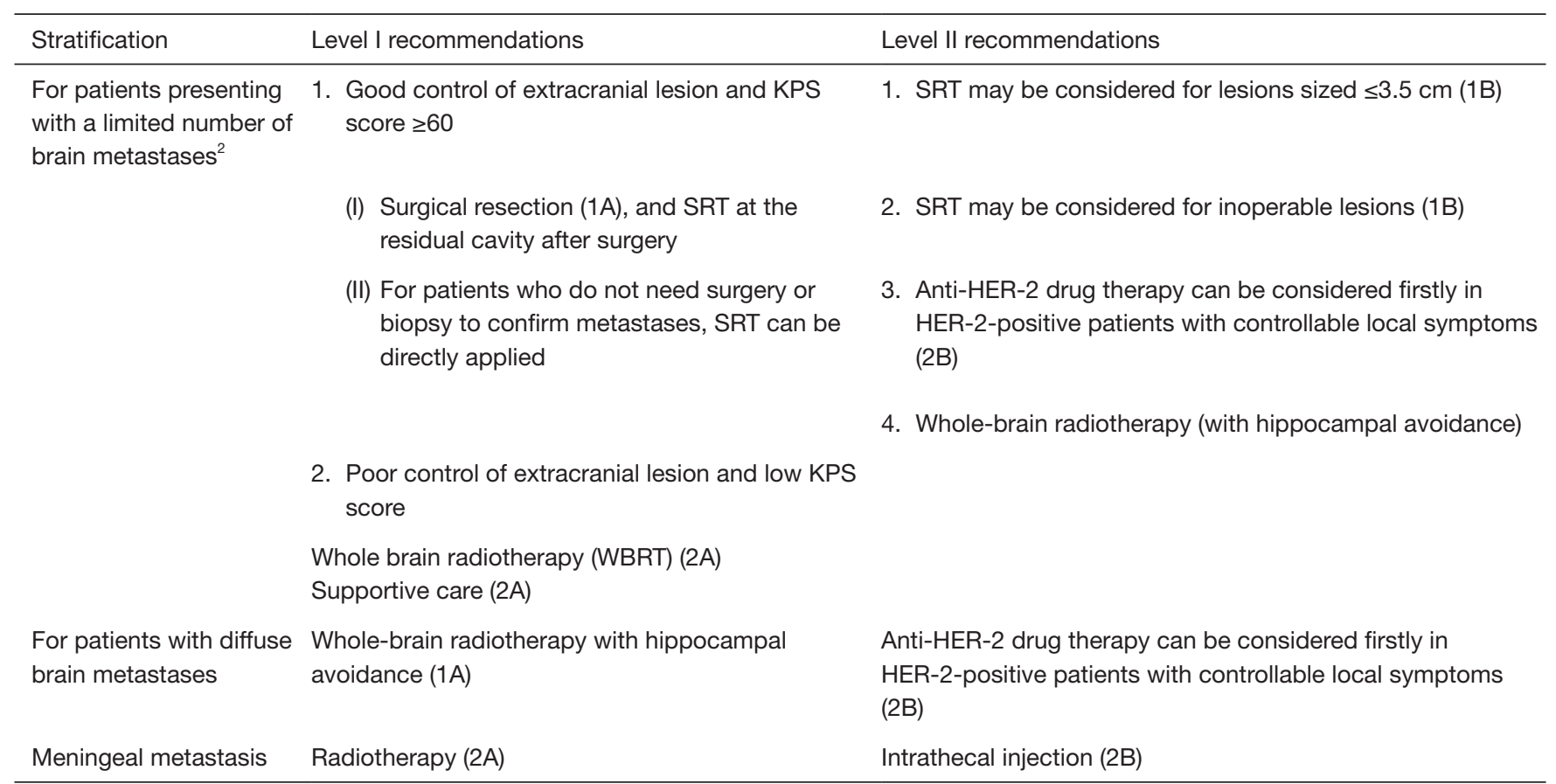

Notes: ${ }^{1}$ The risk of brain metastasis is rising in patient with advanced breast cancer, mainly due to the following two reasons: (I) the availability of more effective systemic treatment of breast cancer has prolonged the survival of patients; and (II) the application of brain MRI has found more patients with asymptomatic brain metastases. The incidence of brain metastasis differs in patients with different types of breast cancer. Generally, the risk of brain metastasis is relatively high in patients with triple-negative breast cancer or HER-2-positive breast cancer, suggesting the importance of monitoring the occurrence of brain metastasis in these patients. In addition, studies have shown that high histological grade and high proliferative activity of the primary tumor, young age, high tumor burden, and BRCA gene mutations are also high-risk factors for brain metastasis. The most common site of brain metastases is the cerebrum. Less often, cancer spreads to the cerebellum and brain stem.

2"A limited number of brain metastases" generally means that the number of metastases is not necessarily limited to 3 or less, but the maximum diameter of a single lesion should not exceed $3 \mathrm{~cm}$; all the lesions can be treated with SRT, and the local control rates are consistent with that of whole-brain radiotherapy.

${ }^{3}$ For patients with recurrence after local treatment of brain metastases, re-operation or SRT may be considered if there is no history of intracranial radiotherapy, the general condition is good, and the extracranial lesions are well controlled. Wholebrain radiotherapy (SRT with hippocampal protection) may also be considered, along with the use of memantine. If the size of the metastasis exceeds the indications of SRT and is not suitable for re-operation, whole-brain radiotherapy can be considered. For patients experience relapse after whole-brain radiotherapy, SRT can be considered. If relapse occurs after SRT, SRT or whole-brain radiotherapy can be repeated. In short, the treatment strategy for recurrence after local treatment of brain metastases should consider the patient's physical condition, the control of extracranial lesions, the patient's quality of 
life, and the possible benefit from treatment.

${ }^{4}$ Many studies have confirmed that less than $10 \%$ of patients presented with brain metastases within $5 \mathrm{~mm}$ of the hippocampus and no patient presented with a metastasis in the hippocampus itself. Therefore, whole-brain radiotherapy with hippocampal avoidance, combined with memantine if appropriate, is recommended for patients with good systemic condition, satisfactory control of extracranial lesions, and a distance of not less than $1 \mathrm{~cm}$ between the lesion and the hippocampus, because such a protocol has high efficiency and low toxicity.

${ }^{5}$ Generally, the effectiveness of medications is far from satisfactory in treating breast cancer brain metastasis. Studies have shown that chemotherapy drugs including capecitabine, topotecan, and temozolomide may achieve certain responses in patients with brain metastases. Other anti-HER-2 small-molecule tyrosine kinase inhibitors such as lenvatinib and pyrotinib have also shown certain activities against brain metastases.

${ }^{6}$ Symptomatic and supportive treatment is one of the main treatments for breast cancer brain metastases as it can improve the quality of life of patients and help the implementation of radiotherapy and drug treatment. For patients with intracranial hypertension, mannitol, glucocorticoids (e.g., dexamethasone), diuretics, and other drugs should be routinely used to alleviate the symptoms of cerebral edema. For patients with intractable brain edema after radiotherapy, bevacizumab may be given to attenuate brain edema. Patients with epilepsy should be treated with antiepileptic drugs.

\section{Circulating tumor markers and next-generation sequencing (NGS)}

\section{Circulating tumor cells (CTCs)}

Tumor assessment offers an important basis for predicting therapeutic response and adjusting treatment plans. Currently, there are two main tumor assessment modes: pathology and imaging. However, pathological evaluation is limited by its invasiveness, and imaging techniques are less sensitive and often have lags in generating reports. CTCs refer to cancer cells that detach from a primary tumor and travel through the bloodstream or lymphatic system to other parts of the body. It can reflect the condition of solid tumors to a certain extent and can be used as a supplementary means for pathological diagnosis, disease monitoring, and molecular sequencing. In addition to dynamic monitoring, CTC can also be used to predict the prognosis. It has been applied in molecular typing and cellular sequencing.

According to the 8th edition of the American Joint Committee on Cancer (AJCC) prognostic staging system, a CTC level of $\geq 1 / 7.5 \mathrm{~mL}$ in early breast cancer suggests a poor prognosis. In addition, Chinese researchers have verified that CTC levels at baseline and after treatment can predict the prognosis of patients with advanced breast cancer. While CTC can play a role in molecular typing, CTC HER-2 also plays a key role in predicting the efficacy of HER-2-targeted therapy. Single-cell RNA sequencing has emerged as an indispensable tool that enables the investigations of the internal mechanisms of tumors at the genome or transcriptome level by using CTC, so as to understand the tumor etiologies and the mechanism of drug resistance and even predict drug resistance. The relevant studies in China have greatly promoted the evolution of CTC from counting to phenotyping and paved the way for the clinical utilities of CTC in predicting therapeutic response and exploring drug resistance.

\section{Circulating tumor DNA (ctDNA)}

Circulating tumor DNA (ctDNA) is composed of free DNA fragments that are released from apoptotic or necrotic tumor cells and CTCs and circulate in blood. These DNA fragments usually bind to proteins to form nucleosomes that travel freely in the circulation. The degradation of ctDNA may be related to liver and kidney metabolism, and its half-life varies greatly depending on the size and structure of different DNA fragments, ranging from 10 minutes to 2 hours.

ctDNA can reflect the tumor load in the body within a short period of time and enable real-time and dynamic monitoring of drug efficacy. As a highly sensitive and specific marker, it can promptly predict disease changes, thus playing a key role in early diagnosis, tumor burden monitoring, therapeutic response prediction, assessment of recurrence/metastasis risks, and prognostic analysis. However, due to the extremely low level of ctDNA in blood, it is still difficult to detect tumor- 
related DNA from a large number of free DNA and blood cells. Meanwhile, currently ctDNA detection can only be performed in highly qualified laboratories, and the expensive equipment and the heterogeneous detection criteria also limit its clinical applications.

\section{Next-generation sequencing (NGS)}

NGS is a DNA sequencing technology that has been developed based on PCR and gene chip technique. It determines DNA sequence by capturing special markers carried by bases during DNA replication. During their replication, DNA fragments must be amplified into the same gene clusters and then replicated in concert, thereby increasing the fluorescence intensity and reading the DNA sequence; as the read length increases, the coordination of gene cluster replication decreases, leading to a decrease in the quality of base sequencing, which strictly limits the read length of NGS (no more than $500 \mathrm{bp}$ ). Therefore, NGS is featured by high throughput and short read length.

NGS has enabled the sequencing of thousands to millions of DNA molecules simultaneously. As a highthroughput technology for DNA sequencing, NGS has revolutionized a variety of fields including personalized medicine, detection of genetic diseases, and clinical diagnosis. It is valuable for early diagnosis, monitoring of therapeutic response and drug resistance, and selection of treatment protocols. In the future, the combined application of CTC, ctDNA, NGS, and even third-generation sequencing technologies will provide better services for cancer patients.

\section{Artificial intelligence (AI)}

AI represents a new direction in the era of precision medicine. Big data, deep learning, advances in computing technology, and new healthcare model have offered opportunities for the development of $\mathrm{AI}$ in medicine. AI has been applied in medical imaging, pathology, and decision support systems.

\section{Intelligent imaging assists tumor diagnosis and response evaluation}

Intelligent imaging technologies have played certain roles in lesion diagnosis, response evaluation, and even molecular type prediction. When applied to distinguish benign and malignant lesions based on plain and enhanced images, the intelligent imaging was only inferior to senior radiologists with 20 years of experience. It was also found that the three-dimensional (3D) imaging information integrating clinical information and dynamically enhanced imaging information can be used as a biomarker to identify molecular subtypes of breast cancer, especially when applied for the prediction of triple-negative breast cancer. $\mathrm{Al}$-assisted diagnosis can help doctors diagnose diseases more quickly and accurately, thus improving the efficiency and accuracy of diagnoses.

\section{AI-based pathology accelerates the qualitative and quantitative identification of tumors}

AI-based pathology has been applied in a variety of tumors (e.g., breast cancer), mainly for cytological screening, differentiation of malignant from benign lesions, morphological quantitative analysis, and histological classification. It was found that the HER-2 scoring results for breast cancer specimens were highly agreed between an AI-based pathology system and human pathologists. In terms of molecular pathology, AI-based analysis of the massive genetic information has become an indispensable element for the development of precision medicine. AIbased pathology reduces the workload of pathologists; more importantly, it makes up for the shortcomings of the subjective analysis performed by human pathologists, improves the qualitative and quantitative identification of tumors, increases the accuracy of pathological diagnoses, and offers patients with personalized treatment opinions and improved cancer prognosis prediction. Thus, it promotes the development of precision pathology.

\section{Intelligent decision-making enriches the clinical decision- making models}

$\mathrm{R} \& \mathrm{D}$ of intelligent decision-making systems is to integrate the learning and analysis capabilities of AI tools and the experience of human experts, thus optimizing decisionmaking process. The CSCO BC collaboration team compared the decision-making performance between an AI system and professional doctors in 2,000 cases (38) and found that the IBM-system Watson for Oncology (WFO) had good feasibility and effectiveness in decision-making for breast cancer treatment. It helped clinicians save time and effort, and its auxiliary applications can further improve the standardization of decision-making by human doctors. Meanwhile, intelligent decision-making systems with 
independent intellectual property rights have also emerged in China. The phase II trial of breast cancer intelligent decision-making based on CSCO BC big data and CSCO $\mathrm{BC}$ guidelines has been completed, and its results showed that the intelligent decision-making system based on CSCO guidelines on breast cancer diagnosis and treatment had good performance in decision-making for different types of breast cancer at different stages. In 2019, the CSCO AI system (39) was officially released, and its nationwide application has dramatically promoted the development of intelligent decision-making systems in China.

AI represents a future. Intelligent systems can not only help clinicians save time and energy but also will further improve the precise diagnosis and treatment of tumors. Therefore, the expert group encourages the implementation of AI-related clinical research and the R\&D of AI systems with independent intellectual property rights in China.

\section{Acknowledgments}

Funding: None.

\section{Footnote}

Conflicts of Interest: The authors have completed the ICMJE uniform disclosure form (available at http://dx.doi. org/10.21037/tbcr-2020-2). ZJ serves as an unpaid Editorin-Chief of Translational Breast Cancer Research. JL serves as an unpaid Managing Editor of Translational Breast Cancer Research. ES, XW, HW, JW, YY, QZ, JC, CG, FJ, QL, YL, YP, TS, and SW serve as the unpaid editorial board members of Translational Breast Cancer Research from Mar 2020 to Feb 2022. The other authors have no conflicts of interest to declare.

Ethical Statement: The authors are accountable for all aspects of the work in ensuring that questions related to the accuracy or integrity of any part of the work are appropriately investigated and resolved.

Open Access Statement: This is an Open Access article distributed in accordance with the Creative Commons AttributionNonCommercial-NoDerivs 4.0 International License (CC BYNC-ND 4.0), which permits the non-commercial replication and distribution of the article with the strict proviso that no changes or edits are made and the original work is properly cited (including links to both the formal publication through the relevant DOI and the license). See: https://creativecommons. org/licenses/by-nc-nd/4.0/.

\section{References}

1. Hurvitz SA, Martin M, Symmans WF, et al. Neoadjuvant trastuzumab, pertuzumab, and chemotherapy versus trastuzumab emtansine plus pertuzumab in patients with HER2-positive breast cancer (KRISTINE): a randomised, open-label, multicentre, phase 3 trial. Lancet Oncol 2018;19:115-26.

2. Gianni L, Pienkowski T, Im YH, et al. 5-year analysis of neoadjuvant pertuzumab and trastuzumab in patients with locally advanced, inflammatory, or early-stage HER2-positive breast cancer (NeoSphere): a multicentre, open-label, phase 2 randomised trial. Lancet Oncol 2016;17:791-800.

3. Slamon D, Eiermann W, Robert N, et al. Adjuvant trastuzumab in HER2-positive breast cancer. N Engl J Med 2011;365:1273-83.

4. Untch M, Jackisch C, Schneeweiss A, et al. NAB-Paclitaxel Improves Disease-Free Survival in Early Breast Cancer: GBG 69-GeparSepto. J Clin Oncol 2019;37:2226-34.

5. von Minckwitz G, Huang CS, Mano MS, et al. Trastuzumab Emtansine for Residual Invasive HER2-Positive Breast Cancer. N Engl J Med 2019;380:617-28.

6. Sikov WM, Berry DA, Perou CM, et al. Impact of the addition of carboplatin and/or bevacizumab to neoadjuvant once-per-week paclitaxel followed by dose-dense doxorubicin and cyclophosphamide on pathologic complete response rates in stage II to III triple-negative breast cancer: CALGB 40603 (Alliance). J Clin Oncol 2015;33:13-21.

7. Von Minckwitz G, Schneeweiss A, Loibl S, et al. Neoadjuvant carboplatin in patients with triple-negative and HER2-positive early breast cancer (GeparSixto; GBG 66): a randomised phase 2 trial. Lancet Oncol 2014;15:747-56.

8. Chan A, Delaloge S, Holmes FA, et al. Neratinib after trastuzumab-based adjuvant therapy in patients with HER2-positive breast cancer (ExteNET): a multicentre, randomised, double-blind, placebo-controlled, phase 3 trial. Lancet Oncol 2016;17:367-77.

9. Jones SE, Collea R, Paul D, et al. Adjuvant docetaxel and cyclophosphamide plus trastuzumab in patients with HER2-amplified early stage breast cancer: a single-group, open-label, phase 2 study. Lancet Oncol 2013;14:1121-8.

10. Tolaney SM, Guo H, Pernas S, et al. Seven-Year FollowUp Analysis of Adjuvant Paclitaxel and Trastuzumab Trial for Node-Negative, Human Epidermal Growth Factor Receptor 2-Positive Breast Cancer. J Clin Oncol 2019;37:1868-75.

11. Cuzick J, Sestak I, Baum M, et al. Effect of anastrozole 
and tamoxifen as adjuvant treatment for early-stage breast cancer: 10-year analysis of the ATAC trial. Lancet Oncol 2010;11:1135-41.

12. Regan MM, Neven P, Giobbie-Hurder A, et al. Assessment of letrozole and tamoxifen alone and in sequence for postmenopausal women with steroid hormone receptorpositive breast cancer: the BIG 1-98 randomised clinical trial at 8.1 years median follow-up. Lancet Oncol 2011;12:1101-8.

13. Francis PA, Pagani O, Fleming GF, et al. Tailoring Adjuvant Endocrine Therapy for Premenopausal Breast Cancer. N Engl J Med 2018;379:122-37.

14. Jiang Z, Wang X. Consideration and discussion on ten hot issues of endocrine therapy for breast cancer. Zhonghua Wai Ke Za Zhi 2015;53:895-900.

15. Pagani O, Regan MM, Walley BA, et al. Adjuvant exemestane with ovarian suppression in premenopausal breast cancer. N Engl J Med 2014;371:107-18.

16. Regan MM, Francis PA, Pagani O, et al. Absolute Benefit of Adjuvant Endocrine Therapies for Premenopausal Women With Hormone Receptor-Positive, Human Epidermal Growth Factor Receptor 2-Negative Early Breast Cancer: TEXT and SOFT Trials. J Clin Oncol 2016;34:2221-31.

17. McCormick B, Winter K, Hudis C, et al. RTOG 9804: a prospective randomized trial for good-risk ductal carcinoma in situ comparing radiotherapy with observation. J Clin Oncol 2015;33:709-15.

18. Correa C, Harris EE, Leonardi MC, et al. Accelerated Partial Breast Irradiation: Executive summary for the update of an ASTRO Evidence-Based Consensus Statement. Pract Radiat Oncol 2017;7:73-9.

19. Hurkmans CW, Borger JH, Rutgers EJ, et al. Quality assurance of axillary radiotherapy in the EORTC AMAROS trial 10981/22023: the dummy run. Radiother Oncol 2003;68:233-40.

20. EBCTCG (Early Breast Cancer Trialists' Collaborative Group), McGale P, Taylor C, et al. Effect of radiotherapy after mastectomy and axillary surgery on 10-year recurrence and 20-year breast cancer mortality: meta-analysis of individual patient data for 8135 women in 22 randomised trials. Lancet 2014;383:2127-35.

21. Wardley AM, Pivot X, Morales-Vasquez F, et al. Randomized phase II trial of first-line trastuzumab plus docetaxel and capecitabine compared with trastuzumab plus docetaxel in HER2-positive metastatic breast cancer. J Clin Oncol 2010;28:976-83.

22. Swain SM, Baselga J, Kim SB, et al. Pertuzumab, trastuzumab, and docetaxel in HER2-positive metastatic breast cancer. N Engl J Med 2015;372:724-34.
23. Yan M, Bian L, Hu X, et al. Pyrotinib plus capecitabine for human epidermal growth factor receptor 2-positive metastatic breast cancer after trastuzumab and taxanes (PHENIX): a randomized, double-blind, placebo-controlled phase 3 study. Transl Breast Cancer Res 2020;1:13.

24. Verma S, Miles D, Gianni L, et al. Trastuzumab emtansine for HER2-positive advanced breast cancer. N Engl J Med 2012;367:1783-91.

25. Cameron D, Casey M, Press M, et al. A phase III randomized comparison of lapatinib plus capecitabine versus capecitabine alone in women with advanced breast cancer that has progressed on trastuzumab: updated efficacy and biomarker analyses. Breast Cancer Res Treat 2008;112:533-43.

26. Mavroudis D, Papakotoulas P, Ardavanis A, et al. Randomized phase III trial comparing docetaxel plus epirubicin versus docetaxel plus capecitabine as first-line treatment in women with advanced breast cancer. Ann Oncol 2010;21:48-54.

27. Jiang ZF, Li JB. Ten hot issues of breast cancer in diagnosis and therapy in 2018. Zhonghua Wai Ke Za Zhi 2018;56:95100.

28. Tutt A, Ellis P, Kilburn L, et al. Abstract S3-01: The TNT trial: A randomized phase III trial of carboplatin (C) compared with docetaxel (D) for patients with metastatic or recurrent locally advanced triple negative or "BRCA1/2" breast cancer (CRUK/07/012). Cancer Res 2015;75:S3-01.

29. Schmid P, Rugo HS, Adams S, et al. Atezolizumab plus nab-paclitaxel as first-line treatment for unresectable, locally advanced or metastatic triple-negative breast cancer (IMpassion130): updated efficacy results from a randomised, double-blind, placebo-controlled, phase 3 trial. Lancet Oncol 2020;21:44-59.

30. Robson M, Ruddy KJ, Im SA, et al. Patient-reported outcomes in patients with a germline BRCA mutation and HER2-negative metastatic breast cancer receiving olaparib versus chemotherapy in the OlympiAD trial. Eur J Cancer 2019;120:20-30.

31. Yuan $\mathrm{P}, \mathrm{Hu} X$, Sun T, et al. Eribulin mesilate versus vinorelbine in women with locally recurrent or metastatic breast cancer: A randomised clinical trial. Eur J Cancer 2019;112:57-65.

32. Zhang P, Sun T, Zhang Q, et al. Utidelone plus capecitabine versus capecitabine alone for heavily pretreated metastatic breast cancer refractory to anthracyclines and taxanes: a multicentre, open-label, superiority, phase 3, randomised controlled trial. Lancet Oncol 2017;18:371-83.

33. Nabholtz JM, Buzdar A, Pollak M, et al. Anastrozole is superior to tamoxifen as first-line therapy for advanced 
breast cancer in postmenopausal women: results of a North American multicenter randomized trial. Arimidex Study Group. J Clin Oncol 2000;18:3758-67.

34. Zhang Q, Shao Z, Shen K, et al. Fulvestrant $500 \mathrm{mg}$ vs $250 \mathrm{mg}$ in postmenopausal women with estrogen receptorpositive advanced breast cancer: a randomized, double-blind registrational trial in China. Oncotarget 2016;7:57301-9.

35. Turner NC, Slamon DJ, Ro J, et al. Overall Survival with Palbociclib and Fulvestrant in Advanced Breast Cancer. N Engl J Med 2018;379:1926-36.

36. Sledge GW Jr, Toi M, Neven P, et al. MONARCH 2: Abemaciclib in Combination With Fulvestrant in Women With HR+/HER2- Advanced Breast Cancer Who Had Progressed While Receiving Endocrine Therapy. J Clin

doi: $10.21037 /$ tbcr-2020-2

Cite this article as: Jiang Z, Song E, Wang X, Wang H, Wang X, Wu J, Yin Y, Zhang Q, Chen J, Chen W, Chen X, Chen Z, Fu P, Geng C, Guo B, Hao C, He Y, Jin F, Liao N, Li N, Liu J, Liu Q, Liu Y, Liu Y, Ma J, Nie J, Pan Y, Song C, Sun T, Wang K, Wang S, Wang T, Wang X, Wang B, Wang S, Wang Y, Xu L, Yan M, Yan Y, Yuan P, Yang J, Zhang F, Zhang J, Zhang S, Zeng X, Li J, Xu F. Guidelines of Chinese Society of Clinical Oncology (CSCO) on Diagnosis and Treatment of Breast Cancer (2020 version). Transl Breast Cancer Res 2020;1:27.
Oncol 2017;35:2875-84.

37. Jiang Z, Li W, Hu X, et al. Tucidinostat plus exemestane for postmenopausal patients with advanced, hormone receptorpositive breast cancer (ACE): a randomised, doubleblind, placebo-controlled, phase 3 trial. Lancet Oncol 2019;20:806-15.

38. Xu F, Sepúlveda MJ, Jiang Z, et al. Artificial Intelligence Treatment Decision Support For Complex Breast CancerAmong Oncologists With Varying Expertise. JCO ClinCancer Inform 2019;3:1-15.

39. Li JB, Jiang ZF. Establishment and its application of Chinese society of clinical oncology artificial intelligence system (CSCO AI). Zhonghua Yi Xue Za Zhi2020;100:411-5. 
Supplementary

\section{Categories of Evidences in CSCO Guidelines}

\begin{tabular}{|c|c|c|c|}
\hline \multicolumn{3}{|c|}{ Characteristics of evidence } & Degree of CSCO expert consensus \\
\hline $1 \mathrm{~A}$ & High & Rigorous meta-analysis and large randomized controlled trial & Unanimous consensus (agree: $\geq 80 \%$ ) \\
\hline $1 \mathrm{~B}$ & High & Rigorous meta analyses and large randomized controlled trials & $\begin{array}{l}\text { Near-unanimous consensus, but with } \\
\text { small controversy (agree: } 60-80 \% \text { ) }\end{array}$ \\
\hline 2B & Slightly low & $\begin{array}{l}\text { Meta-analyses of average quality, small randomized controlled trials, } \\
\text { well-designed large retrospective studies, and case-control studies }\end{array}$ & $\begin{array}{l}\text { Near-uniform consensus, but with } \\
\text { small controversy (agree: } 60-80 \% \text { ) }\end{array}$ \\
\hline 3 & Low & Uncontrolled single-arm trials, case reports, and expert opinions & No consensus and large controversy \\
\hline
\end{tabular}

\section{Recommendation Levels in CsCo Guidelines}

\begin{tabular}{ll}
\hline Recommendation grades & Standards \\
\hline Level I recommendations & Category 1 A evidences and some of category 2A evidences \\
& In general, the CSCO guidelines adopt category 1 evidences and some category 2A evidences with \\
& high expert consensus and good availability in China as Level I recommendations. More specifically, \\
& the Level I recommendations in the CSCO guidelines have the following characteristics: a generally \\
& applicable diagnosis/treatment measure (including well-defined indications) with high availability; it \\
& value in diagnosing/treating a specific tumor is well established, and the relevant product has basically \\
& been listed in the National Medical Insurance Category; the decision on a Level I recommendation is \\
& made mainly according to the clear benefit for patients rather than the changes in commercial medical \\
& insurance policies
\end{tabular}

Level II recommendations

Level III recommendations

Not recommended/disagreed

\section{Category 1B evidences and some of category 2A evidences}

In general, the CSCO guidelines adopt category 1B evidences and some category 2A evidences with relatively low expert consensus or not very good availability in China as Level II recommendations. More specifically, the Level II recommendations in the CSCO guidelines have the following characteristics: high-level evidences obtained from randomized controlled multi-center studies in China or in other countries have demonstrated the efficacy and safety of specific drugs or other therapeutic measures, which, however, have poor availability, cost-effectiveness, and affordability; effective but high-priced drugs or treatments can also be used as Level II recommendations when the anti-tumor value is the main consideration

\section{Category 2B evidences and category 3 evidences}

For diagnosis and treatment methods under investigations and there is no strong evidence to support their use, they can also be referred to as Level III recommendations if uniform expert consensus has been reached

For drugs or medical technologies that have been well demonstrated as not beneficial for patients or even may do harm to patients, with uniform expert consensus, a statement of "not recommend by experts" or "disagreed" if necessary should be made. It can be based on evidences of any category at any level 


\section{Diagnosis and examinations of breast cancer}

\section{Confirmation of early breast cancer}

\begin{tabular}{|c|c|}
\hline Location & Basic principles \\
\hline \multirow{3}{*}{ Assessment of primary tumors } & 2. Bilateral diagnostic mammograms \\
\hline & 3. Ultrasound \\
\hline & 4. Breast magnetic resonance imaging $(\mathrm{MRI})^{1}$ \\
\hline \multirow[t]{3}{*}{ Assessment of regional lymph nodes } & 1. Physical examination \\
\hline & 2. Ultrasound \\
\hline & 3. Core biopsy and fine needle aspiration (FNA) of suspected lesions ${ }^{3}$ \\
\hline \multirow[t]{3}{*}{ Evaluation of distant lesions } & 1. Physical examination \\
\hline & 4. Emission computed tomography ${ }^{6}$ \\
\hline & 5. $\mathrm{PET}_{-} \mathrm{CT}^{7}$ \\
\hline
\end{tabular}

Notes: ${ }^{1}$ Breast MRI is a powerful tool in the staging of breast cancer as it can identify the extent and the multifocality and multicentricity (MFMC) of ipsilateral breast tumors and screen contralateral breast tumors at the initial diagnosis; it is helpful for evaluating the size and location of tumors and the treatment response before and after surgery; it helps to evaluate the soft tissue infiltration of the tumor before establishing a surgical protocol and decide whether a breast-conserving surgery can be performed; finally, it is useful in finding some occult malignancies that are not found in other examinations. Notably, false positives do occur during breast MRI, and a decision of surgery should not be made based on MRI findings alone. Biopsy is recommended for suspicious lesions on MRI.

${ }^{2}$ Pathological examination of the primary tumor and the regional lymph nodes is particularly important before treatment. It is recommended that the imaging-guided core needle puncture can dramatically improve the accuracy of biopsy. An excisional breast biopsy is recommended for scattered calcifications that are difficult to puncture or tumors that are not visible on imaging. For suspicious calcifications in clusters, $\mathrm{X}$-ray-guided excisional biopsy of the lesions with metal wire or radioactive particles can be performed. After the resection, a chest X-ray should be performed again to confirm whether the calcifications have been completely removed.

${ }^{3}$ Pathological examination is suggested for regional lymph nodes that are highly suspected of malignancy. Core biopsy is recommended. FNA can be performed if the lymph nodes are too small to obtain. In addition, the role of FNA of lymph nodes has been well recognized in patients whose primary lesions have been confirmed to be breast cancer.

${ }^{4}$ Chest CT is recommended for patients with confirmed breast cancer, especially if the tumors are diagnosed at an advanced stage and at high risk of recurrence.

${ }^{5}$ It is recommended that abdominal ultrasound can be performed first for confirmed patients, and abdominal CT or MRI may be performed when a visceral metastasis is suspected.

${ }^{6}$ Emission computed tomography (ECT) of bones is the most commonly used method for screening bone metastasis. It is highly sensitive but less specific as it can not display the degree of bone destruction. ECT is recommended only for patients with stage IIIA disease. However, for patients with stage I-IIB disease, ECT should also be performed if they are suspected of bone metastases (e.g., presented with bone pain, pathological fractures, elevated alkaline phosphatase, and/or calcemia).

${ }^{7}$ PET-CT plus conventional staging examinations can effectively assist in diagnosis in patients whose stages can not be judged by routine staging methods or if there are doubts, especially in patients with locally advanced or metastatic lesions. However, PET-CT is not recommended for routine staging in patients with stages I and II breast cancer. 


\section{Patbologic diagnosis}

\begin{tabular}{ll}
\hline Diagnostic approaches & Basic principles \\
\hline Basic pathology ${ }^{1,2}$ & 1. Size \\
& 2. Histopathological type ${ }^{4}$ \\
& $\begin{array}{l}\text { 3. Histological grade } \\
\text { 4. Vascular invasion }\end{array}$ \\
& 5. Accompanying in situ carcinoma \\
& 6. Surgical margin \\
Molecular pathology & 7. Lymph node status \\
(See "Molecular typing" for details) & 1. Detection of ER, PR, HER-2, and Ki-67 in all invasive breast cancer \\
\hline
\end{tabular}

Notes: ${ }^{1}$ Specimens used in histopathological examinations include core needle biopsy specimens, vacuum-assisted minimally invasive biopsy specimens, breast mass resection specimens, breast-conserving surgery specimens, total mastectomy (including simple resection and modified radical mastectomy) specimens, sentinel lymph node biopsy specimens, and axillary lymph node specimens. Please refer to "Standardization for Diagnostic Tumor Pathology (Breast Cancer)" for the detailed explanation on specimen fixation, sampling, and morphological descriptions.

${ }^{2} \mathrm{~A}$ pathology report of invasive breast cancer should include all the content related to the treatment and prognosis of the patient, such as tumor size, histological type, histological grade, presence or absence of vascular invasion, presence or absence of accompanying in situ carcinoma, surgical margins, and lymph node status. If a breast cancer specimen is obtained after treatment, the treatment response should also be evaluated. The pathological diagnosis of a ductal carcinoma in situ (DCIS) should include cytonuclear grade, presence or absence of necrosis, and surgical margins. The evaluation of breast conserving surgery specimens should include the shortest distance between the tumor and the resection margin under microscope. If the resection margin is positive, the type of tumor at the resection margin should be indicated.

${ }^{3}$ When invasive carcinoma co-exists with an in situ carcinoma, the scope and maximum diameter of the invasive foci must be identified.

${ }^{4}$ Histological typing should refer to the WHO Classification of Tumors of the Breast, 2012, and some specific histological types need to be confirmed after immunohistochemical testing. The histological grade of breast cancers can be assessed according to the Nottingham Histologic Score system.

${ }^{5}$ Multigene profiling assay can provide information for clinicopathological classification. Many evidence-based medical data have demonstrated its role in predicting prognosis and evaluating treatment response. At present, the commonly used multigene profiling assays include 21-gene Recurrence Score (RS) Assay (OncotypeDX ${ }^{\circledR}$ ), MammaPrint, PAM-50ROR, EndoPredict, and BreastCancerIndex, which are used for predicting the prognosis of patients with early breast cancer within 5 years after standard treatment. According to international clinical data, 21-gene Recurrence Score (RS) Assay $\left(\right.$ OncotypeDX $\left.{ }^{\circledR}\right)$ is both prognostic and predictive of adjuvant chemotherapy benefit in HR-positive, HER-2-negative, nodenegative $\left(\mathrm{T}_{1-2} \mathrm{~N}_{0} \mathrm{M}_{0}\right)$ patients. However, due to the lack of genetic testing data in Chinese populations, no relevant industry standards or consensus has been available in China. We do not recommend that all patients undergo multigene profiling assay; instead, such assays should be rationally selected based on different risks.

\section{Molecular typing}

\begin{tabular}{|c|c|c|c|c|}
\hline & \multicolumn{4}{|c|}{ Parameters } \\
\hline & HER-2 $2^{1,2}$ & $\mathrm{ER}^{3}$ & $\mathrm{PR}^{3,4}$ & $\mathrm{Ki}-67^{5,6}$ \\
\hline HER-2-positive (HR-negative) & + & - & - & Any \\
\hline HER-2-positive (HR-positive) & + & + & Any & Any \\
\hline Triple-negative $^{8}$ & - & - & - & Any \\
\hline Luminal A & - & + & + and high expression & Low expression ${ }^{7}$ \\
\hline Luminal B (HER-2-negative) & - & + & Low expression or - & High expression ${ }^{7}$ \\
\hline
\end{tabular}

Notes: ${ }^{1}$ Refer to "Guidelines on HER2 Detection in Breast Cancer (2019 Edition)" and "Expert Consensus on Clinical Diagnosis and Treatment of Human Epidermal Growth Factor Receptor 2-positive Breast Cancer 2016" for HER-2 detection. All invasive breast cancers must be tested for HER-2 status. For the purpose of HER-2 detection, Immunohistochemistry (IHC) and in situ hybridization (ISH) must be performed in a well-qualified pathology laboratory. When clinicians have doubts about a patient's previous HER-2 testing results (for example, the testing was performed earlier than the introduction of standardized HER2 testing procedure in China, the testing agency has not been accredited, or the testing agency lacks relevant experience), the HER-2 status should be re-tested. 
${ }^{2}$ Cases with HER2 IHC 3+ or ISH positive are regarded as HER2 positive. More specifically,

Interpretation of the results of HER-2 immunohistochemistry (IHC)

IHC 3+ HER-2-positive

$\mathrm{IHC} 2+$

The HER-2 status is uncertain, and ISH should be performed to further identify HER-2 status

$\mathrm{IHC} 1+/ \mathrm{IHC} 0$

HER-2-negative

Interpretation of the results of ISH for HER-2 status

HER2/CEP17 ratio of $\geq 2.0$ with an average HER-2-positive

HER-2 copy number of $\geq 4.0$ signals/cell

HER2/CEP17 ratio of $<2.0$ with an average HER-2-negative

HER-2 copy number of $<4.0$ signals/cell

HER2/CEP17 ratio of $\geq 2.0$ with an average HER-2 copy number of $\geq 6.0$ signals $/$ cell $^{3}$

It is recommended to increase the number of cells for counting in such cases; if the result remains unchanged, it is classified as FISH positive

HER2/CEP17 ratio of $<2.0$ with an average HER2 copy number of $<6.0$ signals/cell but $\geq 4.0$ signals/cell

In such cases, it is recommended to re-count the signals in at least 20 cell nuclei; if the results change, a more comprehensive judgment can be made based on the findings of these two counting sessions

If the above situation persists, it is important that a note should be made in the FISH report that the judgment of the HER-2 status of such patients should also consider the IHC findings; If the IHC result is $3+$, the cancer is HER2-positive; if the IHC result is $0,1+$, or $2+$, the HER-2 status should be judged as negative

HER2/CEP17 ratio of $\geq 2.0$ with an average HER2 copy number of $<4.0$ signals $/$ cell $^{4}$

It is recommended to increase the number of cells for counting in such cases; if the result remains unchanged, it is classified as FISH negative. It is recommended to made a not in the report

${ }^{3}$ For patients with HER2/CEP17 ratio of $<2.0$ with an average HER-2 copy number of $\geq 6.0$ signals/cell: Research has shown that when an alternative chromosome 17 probe is used to replace CEP17, a considerable proportion of the test results in such cases will be converted to an HER-2/alternative chromosome 17 probe ratio of $>2.0$ with an average HER-2 copy number of $\geq 6.0$ signals/cell. However, more evidences are required for this specific population.

${ }^{4}$ For cases in whom the HER-2/CEP17 ratio is $\geq 2.0$ but the average HER-2 copy number is $<4.0$ signals/cell, no clinical trial has demonstrated that they can benefit from HER-2-targeted therapy. Again, more evidences are required for this specific population.

${ }^{5}$ Detection of estrogen and progesterone receptors refer to "Chinese Guidelines on Immunohistochemical (IHC) Testing of Estrogen and Progesterone Receptors (ER/PgR) in Breast Cancer (2015 Edition)". Hormone receptor status should be tested for all invasive and non-invasive breast cancers. Among ER tests, the value of Estrogen receptor $\alpha$ (ER $\alpha$ ) has been well demonstrated. The use of internationally certified kits/reagents is recommended. The ER or PR status is considered positive if at least $1 \%$ of the cells are stained positive, and the staining intensity and the percentage of positive tumor cells should be reported for positive results.

${ }^{6}$ Experts generally agree that PR is an important prognostic indicator of breast cancer, and it is recommended that a PR cutoff of $20 \%$ can be used to differentiate Luminal A and Luminal B tumors.

${ }^{7}$ All invasive breast carcinomas should be tested for Ki-67, and the percentage of Ki-67-positive cells should be reported. Evaluation of the staining intensity is currently not recommended. For tumors with evenly distributed Ki-67-positive cells, the cells can be counted in only three or more randomly selected high-power fields to obtain an average Ki-67 index; for tumor cells with unevenly distributed positive cells, three or more high-power fields of view can be selected in hot-spot areas. ${ }^{8}$ According to the recommendations from the International Ki67 in Breast Cancer Working Group in 2011, after the entire slice is evaluated, 1,000 invasive cancer cells (no less than 500 cancer cells) should be counted in a representative area. Since it is feasible to count more than 500 cancer cells per slice in daily work, it is recommended to evaluate more than 500 cancer cells as possible when the $\mathrm{Ki}-67$ index is at the critical points of $10 \%$ to $30 \%$ to improve accuracy.

${ }^{9}$ The Ki-67 expression thresholds differ in laboratories with different conditions. Most Chinese experts agree that Ki-67 values $<15 \%$ are defined as "low Ki-67" and $>30 \%$ as "high Ki-67". When Ki-67 expression is between $5 \%$ and $30 \%$, clinical decision-making should be made on the basis of a second pathological consultation or other parameters.

${ }^{10}$ For triple-negative breast cancer at the initial test, standard diagnostic methods should be applied to determine ER, PR, and HER-2 levels again. 


\begin{tabular}{ll}
\hline & Basic principles \\
\hline Tumor-related assessments $^{1}$ & 1. Pathological diagnosis and clinical staging of tumors ${ }^{2.3}$ \\
& 2. Tumor pathological type, histological grade, and molecular characteristics (HER-2, ER, PR, and Ki-67) \\
3. Tumor bed localization ${ }^{4}$ \\
Patient assessments ${ }^{5}$ & 1. Past histories (in particular, treatment-related information) \\
2. Physical examination \\
3. Basic hematological tests \\
4. Tests of the function of major organs (including liver, kidneys, and heart) \\
5. Psychological assessment and counseling \\
6. Fertility consultation for women of child-bearing age
\end{tabular}

Notes: ${ }^{1}$ Neoadjuvant therapy refers to systemic drug treatments such as chemotherapy, endocrine therapy, and targeted therapy performed before surgery. Before treatment, an adequate assessment of the tumor and the systemic conditions is crucial for developing a scientific and reasonable treatment protocol.

${ }^{2}$ Refer to the Eighth Edition of the A7CC Cancer Staging Manual, published by the American Joint Committee on Cancer (AJCC), for the clinical staging of breast cancer. For newly diagnosed patients, appropriate examination methods should be selected for TNM staging. The key information includes the number, location, and size of the mass, regional lymph node status, and distant lesions.

${ }^{3}$ The status of axillary lymph nodes should be identified before neoadjuvant treatment as it will be altered during the neoadjuvant treatment. For patients with clinically negative axillary lymph nodes, sentinel lymph node biopsy can be performed before or after neoadjuvant treatment to determine the status of axillary lymph nodes. For patients with clinically positive axillary lymph nodes, needle biopsy is recommended to confirm the diagnosis; for patients whose axillary lymph nodes turn negative after neoadjuvant therapy, sentinel lymph node biopsy is still controversial due to the false negatives.

${ }^{4}$ Tumor bed localization is recommended for the primary tumor before neoadjuvant treatment. A marker may be placed inside the tumor, or the skin above the tumor surface may be marked, so as to inform the subsequent determination of the scope of surgery and possible breast-conserving opportunities. Markers should also be placed for axillary lymph nodes that are found to be positive in preoperative puncture.

${ }^{5}$ Appropriate examination methods should be adopted to evaluate tumors before and after treatment. In principle, tumor size is evaluated by physical examination and ultrasound at every treatment cycle. The treatment response is evaluated according to the Response Evaluation Criteria In Solid Tumors (RECIST) version 1.1; in principle, the same examination method should be continuously used. 
Commonly used regimens in preoperative treatment of HER-2-positive breast cancer

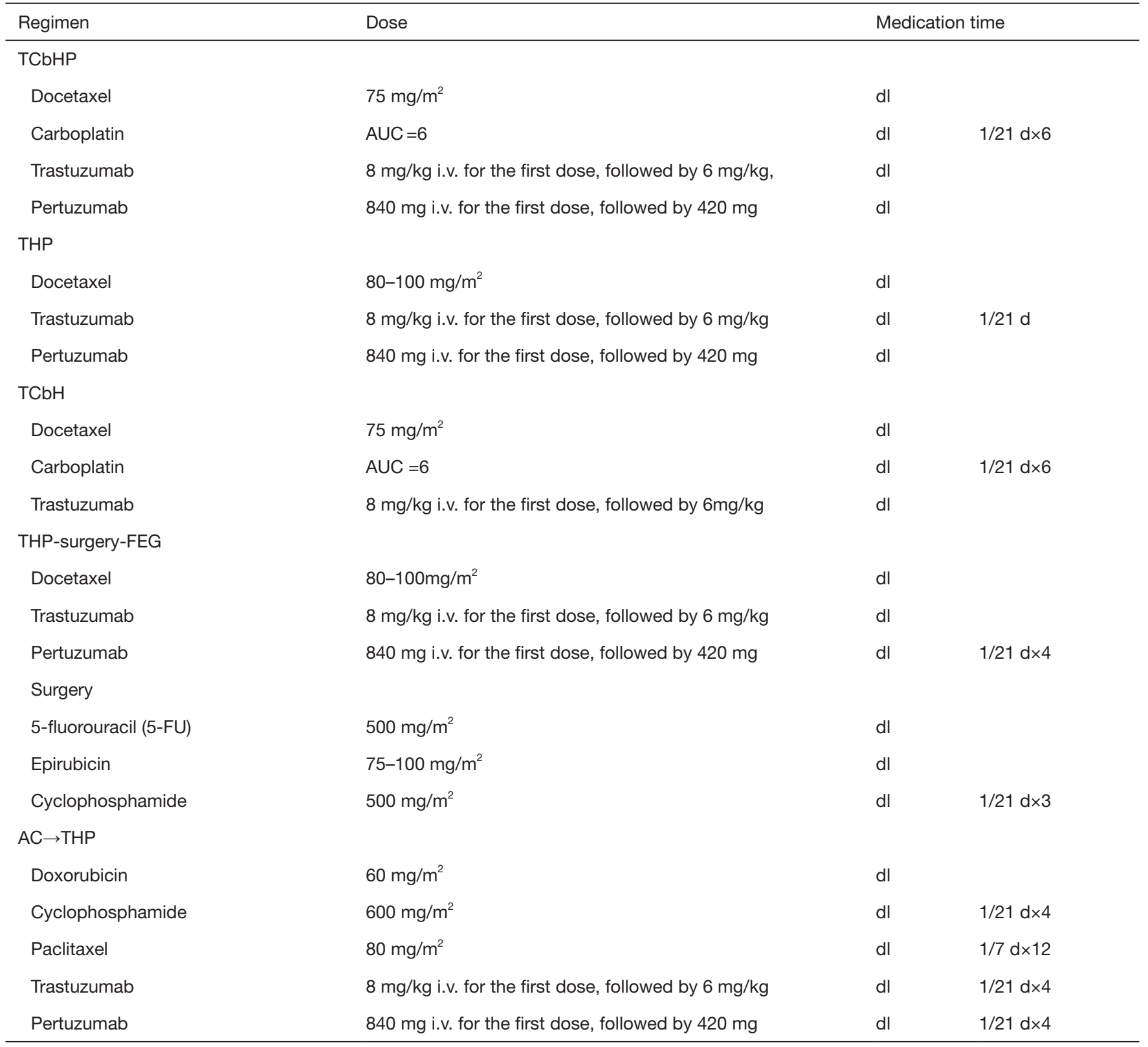


Regimens commonly used as preoperative chemotherapy for HER-2-negative breast cancer

\begin{tabular}{|c|c|c|c|}
\hline Regimen & Dose & \multicolumn{2}{|c|}{ Medication time } \\
\hline Docetaxel & $75 \mathrm{mg} / \mathrm{m}^{2}$ & $\mathrm{dl}$ & $1 / 21 d \times 6$ \\
\hline Doxorubicin & $50 \mathrm{mg} / \mathrm{m}^{2}$ & dl & \\
\hline Cyclophosphamide & $500 \mathrm{mg} / \mathrm{m}^{2}$ & dl & \\
\hline \multicolumn{4}{|c|}{ AT (combination of anthracycline and taxanes) } \\
\hline Doxorubicin & $60 \mathrm{mg} / \mathrm{m}^{2}$ & dl & $1 / 21 d$ \\
\hline or epirubicin & $75 \mathrm{mg} / \mathrm{m}^{2}$ & & \\
\hline Docetaxel & $75 \mathrm{mg} / \mathrm{m}^{2}$ & dl & \\
\hline \multicolumn{4}{|c|}{ AC (anthracycline combined with cyclophosphamide) $\rightarrow T$ (taxanes) } \\
\hline \multicolumn{4}{|c|}{ Anthracycline + cyclophosphamide followed by weekly paclitaxel } \\
\hline Doxorubicin & $60 \mathrm{mg} / \mathrm{m}^{2}$ & & \\
\hline or epirubicin & $100 \mathrm{mg} / \mathrm{m}^{2}$ & dl & $1 / 21 d \times 4$ \\
\hline Cyclophosphamide & $600 \mathrm{mg} / \mathrm{m}^{2}$ & dl & \\
\hline Paclitaxel & $80 \mathrm{mg} / \mathrm{m}^{2}$ & $\mathrm{dl}$ & $1 / 7 d \times 12$ \\
\hline \multicolumn{4}{|c|}{ Anthracycline + cyclophosphamide followed by docetaxel } \\
\hline Doxorubicin & $60 \mathrm{mg} / \mathrm{m}^{2}$ & & \\
\hline or epirubicin & $100 \mathrm{mg} / \mathrm{m}^{2}$ & $\mathrm{dl}$ & $1 / 21 d \times 4$ \\
\hline Cyclophosphamide & $600 \mathrm{mg} / \mathrm{m}^{2}$ & $\mathrm{dl}$ & \\
\hline Docetaxel & $80-100 \mathrm{mg} / \mathrm{m}^{2}$ & dl & $1 / 21 d \times 4$ \\
\hline \multicolumn{4}{|c|}{ AT-NP (anthracycline combined with taxanes - platinum) } \\
\hline Doxorubicin & $60 \mathrm{mg} / \mathrm{m}^{2}$ & & \\
\hline or epirubicin & $75 \mathrm{mg} / \mathrm{m}^{2}$ & $\mathrm{dl}$ & $1 / 21 d \times 4$ \\
\hline Docetaxel & $75 \mathrm{mg} / \mathrm{m}^{2}$ & dl & \\
\hline Vinorelbine & $25 \mathrm{mg} / \mathrm{m}^{2}$ & Days 1,8 & $1 / 21 d \times 4$ \\
\hline Cisplatin & $75 \mathrm{mg} / \mathrm{m}^{2}$ & days $1-3$ & \\
\hline \multicolumn{4}{|c|}{ TP (taxanes combined with platinum) } \\
\hline Albumin paclitaxel & $125 \mathrm{mg} / \mathrm{m}^{2}$ & Days 1,8 & $1 / 21 d \times 6$ \\
\hline Cisplatin & $75 \mathrm{mg} / \mathrm{m}^{2}$ & Days $1-3$ & \\
\hline \multicolumn{4}{|c|}{ TP (taxanes combined with platinum) } \\
\hline Albumin paclitaxel & $125 \mathrm{mg} / \mathrm{m}^{2}$ & Days 1,8 & $1 / 21 d \times 6$ \\
\hline Carboplatin & $A \cup C=6$ & $\mathrm{dl}$ & \\
\hline
\end{tabular}

Note: Avoid myelosuppression during chemotherapy. Use of colony-stimulating factor (CSF) for prophylaxis is recommended before commencement of chemotherapy regimens (see "Management of therapy-induced disorders" for details). 
Assessment and examinations before adjuvant treatment

\begin{tabular}{|c|c|}
\hline & Basic principles \\
\hline \multirow{2}{*}{ Tumor-related assessments } & 2. Tumor pathological type, histological grade, and molecular characteristics (HER-2, ER, PR, and Ki-67) ${ }^{2}$ \\
\hline & $\begin{array}{l}\text { 3. Multigene profiling assays }{ }^{3} \text { including } 21 \text {-gene Recurrence Score (RS) Assay (OncotypeDX }{ }^{\circledR} \text { ) and } \\
70 \text {-gene assay (MammaPrint }{ }^{\circledR} \text { ). }\end{array}$ \\
\hline \multirow{3}{*}{ Patient assessments ${ }^{4}$} & 3. Basic hematological tests \\
\hline & 4. Tests of the function of major organs (including liver, kidneys, and heart) \\
\hline & 5. Psychological assessment and counseling \\
\hline
\end{tabular}

Notes: ${ }^{1}$ The clinical staging of tumors is based on the Eighth Edition of American Foint Committee on Cancer (AfCC) Cancer Staging Manual. For postoperative patients, TNM staging should be performed based on the pathological findings including the number, location, and size of the mass, regional lymph node status, and resection margins.

${ }^{2}$ See "Pathologic diagnosis" and "Molecular typing" for details.

${ }^{3}$ Foreign guidelines recommend gene expression determination as an important basis for selecting adjuvant chemotherapy for some HR-positive and HER-2 negative patients. However, there are still few researches on gene expression determination in Chinese populations, and there is a lack of corresponding industry standards and consensus in China. Therefore, the test populations should be chosen with caution.

${ }^{4}$ The patients' general conditions as well as their tolerance to treatment should be assessed carefully, so as inform the development of a multidisciplinary treatment protocol. 
Commonly used regimens in adjuvant therapy for HER-2-positive breast cancer

\begin{tabular}{|c|c|c|c|}
\hline Regimen & Dose & Medication time & Cycle and duration \\
\hline \multicolumn{4}{|c|}{ AC (anthracycline combined with cyclophosphamide) $\rightarrow$ THP (taxanes combined with trastuzumab and pertuzumab) } \\
\hline \multicolumn{4}{|c|}{ Doxorubicin + cyclophosphamide followed by paclitaxel + trastuzumab + pertuzumab } \\
\hline Doxorubicin & $60 \mathrm{mg} / \mathrm{m}^{2}$ & d1 & \\
\hline Cyclophosphamide & $600 \mathrm{mg} / \mathrm{m}^{2}$ & d1 & $1 / 21 d \times 4$ \\
\hline Paclitaxel & $80 \mathrm{mg} / \mathrm{m}^{2}$ & d1 & $1 / 7 \mathrm{~d} \times 12$ \\
\hline Trastuzumab & $8 \mathrm{mg} / \mathrm{kg}$ i.v. for the first dose, followed by $6 \mathrm{mg} / \mathrm{kg}$ & d1 & \multirow{2}{*}{$1 / 21 d$, for one year } \\
\hline Pertuzumab & $840 \mathrm{mg}$ i.v. for the first dose, followed by $420 \mathrm{mg}$ & d1 & \\
\hline \multicolumn{4}{|c|}{ Doxorubicin + cyclophosphamide followed by docetaxel + trastuzumab + pertuzumab } \\
\hline Doxorubicin & $60 \mathrm{mg} / \mathrm{m}^{2}$ & d1 & \\
\hline Cyclophosphamide & $600 \mathrm{mg} / \mathrm{m}^{2}$ & d1 & $1 / 21 d \times 4$ \\
\hline Docetaxel & $80-100 \mathrm{mg} / \mathrm{m}^{2}$ & d1 & $1 / 21 d \times 4$ \\
\hline Trastuzumab & $8 \mathrm{mg} / \mathrm{kg}$ i.v. for the first dose, followed by $6 \mathrm{mg} / \mathrm{kg}$ & d1 & \multirow{2}{*}{$1 / 21 \mathrm{~d}$, for one year } \\
\hline Pertuzumab & $840 \mathrm{mg}$ i.v. for the first dose, followed by $420 \mathrm{mg}$ & d1 & \\
\hline \multicolumn{4}{|l|}{ TCbHP } \\
\hline Docetaxel & $75 \mathrm{mg} / \mathrm{m}^{2}$ & d1 & \\
\hline Carboplatin & $\mathrm{AUC}=6$ & d1 & $1 / 21 d \times 6$ \\
\hline Trastuzumab & $8 \mathrm{mg} / \mathrm{kg}$ i.v. for the first dose, followed by $6 \mathrm{mg} / \mathrm{kg}$ & d1 & \\
\hline Pertuzumab & $840 \mathrm{mg}$ i.v. for the first dose, followed by $420 \mathrm{mg}$ & d1 & $1 / 21 d$, for one year \\
\hline \multicolumn{4}{|c|}{ Anthracycline + cyclophosphamide $\rightarrow$ sequential docetaxel + trastuzumab } \\
\hline $\begin{array}{l}\text { Doxorubicin } \\
\text { or epirubicin }\end{array}$ & $\begin{array}{l}60 \mathrm{mg} / \mathrm{m}^{2} \\
100 \mathrm{mg} / \mathrm{m}^{2}\end{array}$ & d1 & $1 / 21 d \times 4$ \\
\hline Cyclophosphamide & $600 \mathrm{mg} / \mathrm{m}^{2}$ & d1 & \\
\hline Docetaxel & $80-100 \mathrm{mg} / \mathrm{m}^{2}$ & d1 & $1 / 21 d \times 4$ \\
\hline Trastuzumab & $8 \mathrm{mg} / \mathrm{kg}$ i.v. for the first dose, followed by $6 \mathrm{mg} / \mathrm{kg}$ & d1 & $1 / 21 d$, for one year \\
\hline \multicolumn{4}{|c|}{ Anthracycline + cyclophosphamide $\rightarrow$ sequential paclitaxel + trastuzumab } \\
\hline Doxorubicin & $60 \mathrm{mg} / \mathrm{m}^{2}$ & d1 & \\
\hline or epirubicin & $100 \mathrm{mg} / \mathrm{m}^{2}$ & & $1 / 21 d \times 4$ \\
\hline Cyclophosphamide & $600 \mathrm{mg} / \mathrm{m}^{2}$ & d1 & \\
\hline Paclitaxel & $80 \mathrm{mg} / \mathrm{m}^{2}$ & d1 & $1 / 7 \mathrm{~d} \times 12$ \\
\hline Trastuzumab & $4 \mathrm{mg} / \mathrm{kg}$ i.v. for the first dose, followed by $2 \mathrm{mg} / \mathrm{kg}$ & d1 & $1 / 7 \mathrm{~d}$, for one year \\
\hline \multicolumn{4}{|c|}{ Dose-dense doxorubicin + cyclophosphamide $\rightarrow$ paclitaxel + trastuzumab } \\
\hline Doxorubicin & $60 \mathrm{mg} / \mathrm{m}^{2}$ & d1 & \\
\hline Cyclophosphamide & $600 \mathrm{mg} / \mathrm{m}^{2}$ & d1 & $1 / 14 d \times 4$ \\
\hline Paclitaxel & $175 \mathrm{mg} / \mathrm{m}^{2}$ & d1 & $1 / 14 d \times 4$ \\
\hline Trastuzumab & $4 \mathrm{mg} / \mathrm{kg}$ i.v. for the first dose, followed by $2 \mathrm{mg} / \mathrm{kg}$ & d1 & $1 / 7 \mathrm{~d}$, for one year \\
\hline \multicolumn{4}{|l|}{$\mathrm{TCbH}$} \\
\hline Docetaxel & $75 \mathrm{mg} / \mathrm{m}^{2}$ & d1 & \\
\hline Carboplatin & $\mathrm{A} \cup \mathrm{C}=6$ & d1 & $1 / 21 d \times 6$ \\
\hline Trastuzumab & $8 \mathrm{mg} / \mathrm{kg}$ i.v. for the first dose, followed by $6 \mathrm{mg} / \mathrm{kg}$ & d1 & $1 / 21 d$, for one year \\
\hline \multicolumn{4}{|l|}{$\mathrm{TC}+\mathrm{H}$} \\
\hline Docetaxel & $75 \mathrm{mg} / \mathrm{m}^{2}$ & d1 & $1 / 21 d \times 4$ \\
\hline Cyclophosphamide & $600 \mathrm{mg} / \mathrm{m}^{2}$ & d1 & \\
\hline Trastuzumab & $8 \mathrm{mg} / \mathrm{kg}$ i.v. for the first dose, followed by $6 \mathrm{mg} / \mathrm{kg}$ & d1 & $1 / 21 d$, for one year \\
\hline \multicolumn{4}{|c|}{ TH (weekly paclitaxel + trastuzumab) } \\
\hline Paclitaxel & $80 \mathrm{mg} / \mathrm{m}^{2}$ & d1 & $1 / 7 d \times 12$ \\
\hline Trastuzumab & $4 \mathrm{mg} / \mathrm{kg}$ i.v. for the first dose, followed by $2 \mathrm{mg} / \mathrm{kg}$ & d1 & $1 / 7 \mathrm{~d}$, for one year \\
\hline
\end{tabular}


Regimens commonly used as adjuvant chemotherapy for HER-2-negative breast cancer

\begin{tabular}{|c|c|c|c|}
\hline Regimen & Dose & Medication time & Cycle and duration \\
\hline \multicolumn{4}{|c|}{ AC (anthracycline combined with cyclophosphamide) $\rightarrow T$ (sequential taxanes) } \\
\hline \multicolumn{4}{|c|}{ Anthracycline + cyclophosphamide - followed by docetaxel } \\
\hline Doxorubicin & $60 \mathrm{mg} / \mathrm{m}^{2}$ & & \\
\hline or epirubicin & $100 \mathrm{mg} / \mathrm{m}^{2}$ & d1 & $1 / 21 d \times 4$ \\
\hline Cyclophosphamide & $600 \mathrm{mg} / \mathrm{m}^{2}$ & d1 & \\
\hline Docetaxel & $80-100 \mathrm{mg} / \mathrm{m}^{2}$ & d1 & $1 / 21 d \times 4$ \\
\hline \multicolumn{4}{|c|}{ Anthracycline + cyclophosphamide $\rightarrow$ followed by weekly paclitaxel } \\
\hline Doxorubicin & $60 \mathrm{mg} / \mathrm{m}^{2}$ & & \\
\hline or epirubicin & $100 \mathrm{mg} / \mathrm{m}^{2}$ & d1 & $1 / 21 d \times 4$ \\
\hline Cyclophosphamide & $600 \mathrm{mg} / \mathrm{m}^{2}$ & d1 & \\
\hline Paclitaxel & $80 \mathrm{mg} / \mathrm{m}^{2}$ & d1 & $1 / 7 d \times 12$ \\
\hline \multicolumn{4}{|c|}{ Dose-dense anthracycline + cyclophosphamide $\rightarrow$ dose-dense paclitaxel } \\
\hline Doxorubicin & $60 \mathrm{mg} / \mathrm{m}^{2}$ & d1 & \\
\hline or epirubicin & $100 \mathrm{mg} / \mathrm{m}^{2}$ & & $1 / 14 \mathrm{~d} \times 4$ \\
\hline Cyclophosphamide & $600 \mathrm{mg} / \mathrm{m}^{2}$ & d1 & \\
\hline Paclitaxel & $175 \mathrm{mg} / \mathrm{m}^{2}$ & d1 & $1 / 14 \mathrm{~d} \times 4$ \\
\hline \multicolumn{4}{|c|}{ Dose-dense anthracycline + cyclophosphamide $\rightarrow$ weekly paclitaxel } \\
\hline Doxorubicin & $60 \mathrm{mg} / \mathrm{m}^{2}$ & & \\
\hline or epirubicin & $100 \mathrm{mg} / \mathrm{m}^{2}$ & d1 & $1 / 14 \mathrm{~d} \times 4$ \\
\hline Cyclophosphamide & $600 \mathrm{mg} / \mathrm{m}^{2}$ & d1 & \\
\hline Paclitaxel & $80 \mathrm{mg} / \mathrm{m}^{2}$ & d1 & $1 / 7 d \times 12$ \\
\hline \multicolumn{4}{|c|}{ AC (anthracyclines + cyclophosphamide) } \\
\hline Doxorubicin & $60 \mathrm{mg} / \mathrm{m}^{2}$ & & \\
\hline or epirubicin & $100 \mathrm{mg} / \mathrm{m}^{2}$ & d1 & $1 / 21 d \times 4$ \\
\hline Cyclophosphamide & $600 \mathrm{mg} / \mathrm{m}^{2}$ & d1 & \\
\hline TC & & & $1 / 21 d \times 4$ \\
\hline Docetaxel & $75 \mathrm{mg} / \mathrm{m}^{2}$ & d1 & \\
\hline Cyclophosphamide & $600 \mathrm{mg} / \mathrm{m}^{2}$ & d1 & \\
\hline TAC & & & $1 / 21 d \times 6$ \\
\hline Docetaxel & $75 \mathrm{mg} / \mathrm{m}^{2}$ & d1 & \\
\hline Adriamycin & $50 \mathrm{mg} / \mathrm{m}^{2}$ & d1 & \\
\hline Cyclophosphamide & $500 \mathrm{mg} / \mathrm{m}^{2}$ & d1 & \\
\hline \multicolumn{4}{|l|}{$\mathrm{FEC} \rightarrow \mathrm{T}$} \\
\hline 5-fluorouracil (5-FU) & $500 \mathrm{mg} / \mathrm{m}^{2}$ & d1 & \\
\hline Epirubicin & $100 \mathrm{mg} / \mathrm{m}^{2}$ & d1 & $1 / 21 d \times 3$ \\
\hline Cyclophosphamide & $500 \mathrm{mg} / \mathrm{m}^{2}$ & d1 & \\
\hline Docetaxel & $80-100 \mathrm{mg} / \mathrm{m}^{2}$ & d1 & $1 / 21 d \times 3$ \\
\hline \multicolumn{4}{|l|}{ FAC } \\
\hline 5-fluorouracil (5-FU) & $500 \mathrm{mg} / \mathrm{m}^{2}$ & Days 1 and 8 & \\
\hline Doxorubicin & $500 \mathrm{mg} / \mathrm{m}^{2}$ & d1 & $1 / 21 d \times 6$ \\
\hline Cyclophosphamide & $500 \mathrm{mg} / \mathrm{m}^{2}$ & d1 & \\
\hline
\end{tabular}

Note: Avoid myelosuppression during chemotherapy. Use of colony-stimulating factor (CSF) for prophylaxis is recommended before commencement of chemotherapy regimens (see "Management of therapy-induced disorders" for details). 


\begin{tabular}{|c|c|}
\hline & Basic principles \\
\hline \multirow[t]{5}{*}{ Assessment of general conditions } & 1. Previous medical history ${ }^{1}$ \\
\hline & 2. Physical examination \\
\hline & 3. Hematology \\
\hline & 4. Tests of the function of major organs (including liver, kidneys, and heart ${ }^{2}$ ) \\
\hline & 5. Psychological assessment and counseling \\
\hline \multirow[t]{8}{*}{ Examinations for confirmation of the diagnosis } & 1. Pathology consultation of primary lesion ${ }^{3}$ \\
\hline & 2. Pathological biopsy of metastatic lesions ${ }^{3}$ \\
\hline & 3. Chest CT \\
\hline & 4. Abdomen ultrasound ${ }^{4}$ \\
\hline & 5. Pelvic ultrasound ${ }^{4}$ \\
\hline & 6. Bone scan ${ }^{5}$ \\
\hline & 7. Screening imaging and further examinations of suspicious metastases ${ }^{6,7}$ \\
\hline & 8. $\mathrm{PET}-\mathrm{CT}^{7}$ \\
\hline
\end{tabular}

Notes: ${ }^{1}$ Inquire the patient's prior treatment histories, which include patients' preoperative neoadjuvant treatment, postoperative adjuvant treatment, and treatment in recurrence and metastasis stages. The key information includes: (I) chemotherapy: regimens, doses, cycles, treatment responses, and reasons for drug discontinuation; (II) endocrine therapy: drugs, doses, treatment responses, and reasons for discontinuation; and (III) radiotherapy: target regions, therapeutic rays, doses, and efficacy.

${ }^{2}$ Patients who are planning to be treated with anthracyclines, trastuzumab, and pertuzumab, especially the elderly patients with medical histories such as high blood pressure and cardiovascular disease, should undergo adequate cardiac function assessment before treatment, so as to decide the use (or not) of these therapies and optimize the drugs and dosages.

${ }^{3}$ For patients experiencing recurrence and/or metastasis, the pathological condition of the primary lesion should be reconfirmed as possible. Re-biopsy of the recurrent and/or metastasis lesion can be performed if necessary. Re-examinations are particularly important if: (I) the previous ER, PR, and HER-2 statuses of the primary tumor are unknown; (II) the test results are negative; and/or (III) HER-2 ICH 2+ but FISH has not been performed.

${ }^{4}$ For patients with suspicious lesions on the abdominal and/or pelvic ultrasound, CT or MRI of the abdomen and pelvis is recommended.

${ }^{5}$ Emission computed tomography (ECT) of bones is the most commonly used method for screening bone metastasis. X-ray, CT, or MRI of symptomatic bones and long bones and weight-bearing bones with abnormal ECT findings is recommended to further confirm the diagnosis for the diagnosis and treatment of breast cancer bone metastases and bone-related diseases.

${ }^{6} \mathrm{CT}$ or MRI scan of the head should be performed in patients presenting with symptoms or signs of the central nervous system. Brain imaging should be performed on a regular basis for some high-risk postoperative patients with asymptomatic triple-negative, HER-2-positive breast cancer or those with rapid progression of recurrent and metastatic disease.

${ }^{7}$ PET-CT helps to find metastatic lesions in other organs and tissues. PET-CT can also be considered when there is a need to identify multifocal breast cancer. However, in patients with recurrent and metastatic breast cancer, CT or MRI is still required for baseline examination before treatment, so as to facilitate the evaluation of treatment response in the future. Routine use of PET-CT in the assessment of treatment response is not recommended.

${ }^{8}$ The treatment response of patients with advanced breast cancer should be evaluated regularly according to Response Evaluation Criteria in Solid Tumors (RECIST) version 1.1. The evaluation cycle and response evaluation should also be based on the changes in disease conditions/symptoms and the therapies. Generally, safety evaluation (including hematology) should be performed every cycle, along with the evaluation of the treatment tolerance. In principle, imaging examinations of target lesions should be performed every two cycles.

${ }^{9}$ During the treatment of advanced breast cancer, tumor markers are auxiliary indicators for evaluating treatment response, and their dynamic changes can help judge changes in disease condition. Elevated tumor markers may either reflect tumor progression or be just a transient state after effective treatment. Therefore, a second test for tumor markers should be performed one month later, and a decision of modifying the treatment protocol (or not) should be made according to the patient's symptoms and imaging findings. Notably, elevated tumor markers alone cannot be used as a basis for changing/ modifying the treatment protocol. 
Trastuzumab-containing regimens for recurrent/metastatic breast cancer

\begin{tabular}{|c|c|c|c|}
\hline Regimen & Dose & Medication time & Cycle and duration \\
\hline \multicolumn{4}{|c|}{ THP (taxanes combined with trastuzumab and pertuzumab) } \\
\hline Docetaxel & $75 \mathrm{mg} / \mathrm{m}^{2}$ & d1 & $1 / 21 d$ \\
\hline Or albumin paclitaxel & $100-150 \mathrm{mg} / \mathrm{m}^{2}$ & d1 1 & $1 / 7 d$ \\
\hline Or paclitaxel & $80 \mathrm{mg} / \mathrm{m}^{2}$ & d1 & $1 / 7 d$ \\
\hline Pertuzumab & $840 \mathrm{mg}$ initial dose, followed by $420 \mathrm{mg}$ & d1 1 & $1 / 21 d$ \\
\hline \multicolumn{4}{|l|}{$\mathrm{TXH}$} \\
\hline Docetaxel & $75 \mathrm{mg} / \mathrm{m}^{2}$ & d1 & $1 / 21 d$ \\
\hline Capecitabine & $1,000 \mathrm{mg} / \mathrm{m}^{2}$, b.i.d. & Days $1-14$ & $1 / 21 d$ \\
\hline \multicolumn{4}{|c|}{ TH (taxanes combined with trastuzumab) } \\
\hline Albumin paclitaxel & $100-150 \mathrm{mg} / \mathrm{m}^{2}$ & d1 & $1 / 7 d$ \\
\hline Trastuzumab & $4 \mathrm{mg} / \mathrm{kg}$ initial dose, followed by $2 \mathrm{mg} / \mathrm{kg}$ & d1 & $1 / 7 d$ \\
\hline \multicolumn{4}{|c|}{ Docetaxel combined with trastuzumab } \\
\hline Docetaxel & $75 \mathrm{mg} / \mathrm{m}^{2}$ & d1 & $1 / 21 d$ \\
\hline Trastuzumab & $8 \mathrm{mg} / \mathrm{kg}$ initial dose, followed by $6 \mathrm{mg} / \mathrm{kg}$ & d1 & $1 / 21 d$ \\
\hline \multicolumn{4}{|l|}{$\mathrm{NH}$} \\
\hline Vinorelbine & $25 \mathrm{mg} / \mathrm{m}^{2}$ & d1 & $1 / 7 d$ \\
\hline
\end{tabular}

Regimens after trastuzumab has failed

\begin{tabular}{|c|c|c|c|}
\hline Regimen & Dose & Medication time & Cycle and duration \\
\hline \multicolumn{4}{|c|}{ Pyrotinib + capecitabine } \\
\hline Pyrotinib & $400 \mathrm{mg}$ & Daily & Daily \\
\hline Capecitabine & $1,000 \mathrm{mg} / \mathrm{m}^{2}$, b.i.d. & Days 1-14 & $1 / 21 d$ \\
\hline \multicolumn{4}{|c|}{ Lapatinib + capecitabine } \\
\hline Lapatinib & $1,250 \mathrm{mg}$ & Daily & Daily \\
\hline Capecitabine & $1,000 \mathrm{mg} / \mathrm{m}^{2}$, b.i.d. & Days 1-14 & $1 / 21 d$ \\
\hline \multicolumn{4}{|l|}{ T-DM1 } \\
\hline T-DM1 & $3.6 \mathrm{mg} / \mathrm{kg}$ & d1 & $1 / 21 d$ \\
\hline \multicolumn{4}{|c|}{ Lapatinib + trastuzumab } \\
\hline Lapatinib & $1,250 \mathrm{mg}$ & Daily & Daily \\
\hline Trastuzumab & $8 \mathrm{mg} / \mathrm{kg}$ initial dose, followed by $6 \mathrm{mg} / \mathrm{kg}$ & d1 & $1 / 21 d$ \\
\hline
\end{tabular}


Commonly used single-agent chemotherapy regimens for recurrent or metastatic breast cancer

\begin{tabular}{llll}
\hline Regimen & Dose & Medication time & Cycle and duration \\
\hline Albumin paclitaxel & $100-150 \mathrm{mg} / \mathrm{m}^{2}$ & $\mathrm{~d} 1$ & $\mathrm{l} / 7 \mathrm{~d}$ \\
Docetaxel & $75 \mathrm{mg} / \mathrm{m}^{2}$ & $\mathrm{~d} 1$ & $1 / 21 \mathrm{~d}$ \\
Paclitaxel & $80 \mathrm{mg} / \mathrm{m}^{2}$ & $\mathrm{~d} 1$ & $\mathrm{l} / 7 \mathrm{~d}$ \\
Capecitabine & $1,000 \mathrm{mg} / \mathrm{m}^{2}$, b.i.d. & Days $1-14$ & $1 / 21 \mathrm{~d}$ \\
Gemcitabine & $1,000 \mathrm{mg} / \mathrm{m}^{2}$ & Days 1 and 8 or days 1,8, & $1 / 21 \mathrm{~d}$ \\
& & and 15 & $1 / 28 \mathrm{~d}$ \\
Vinorelbine & $25 \mathrm{mg} / \mathrm{m}^{2}$ or vinorelbine (soft capsule: initial & Days 1 and 8 or days 1,8, & $1 / 21 \mathrm{~d}$ \\
& dose of $60 \mathrm{mg} / \mathrm{m}^{2}$ for three weeks, followed by & and 15 & $1 / 28 \mathrm{~d}$ \\
Epirubicin & $80 \mathrm{mg} / \mathrm{m}^{2}$ if well tolerated & & $1 / 21 \mathrm{~d}$ \\
Doxorubicin & $60-90 \mathrm{mg} / \mathrm{m}^{2}$ & $\mathrm{~d} 11$ & $1 / 21 \mathrm{~d}$ \\
Eribulin & $50 \mathrm{mg} / \mathrm{m}^{2}$ & $\mathrm{~d} 11$ & $1 / 21 \mathrm{~d}$ \\
Liposomal doxorubicin & $1.4 \mathrm{mg} / \mathrm{m}^{2}$ & Days 1 and 8 & $1 / 21 \mathrm{~d}$ \\
Liposomal paclitaxel & $30-50 \mathrm{mg} / \mathrm{m}^{2}$ & $\mathrm{~d} 1 \mathrm{~d}$ & $1 / 21 \mathrm{~d}$ \\
\hline
\end{tabular}

Commonly used combination chemotherapy regimens for recurrent or metastatic breast cancer

\begin{tabular}{|c|c|c|c|}
\hline Regimen & Dose & Medication time & Cycle and duration \\
\hline \multicolumn{4}{|c|}{ TX (taxanes combined with capecitabine) } \\
\hline Docetaxel & $75 \mathrm{mg} / \mathrm{m}^{2}$ & d1 & $1 / 21 d$ \\
\hline Or albumin paclitaxel & $100-150 \mathrm{mg} / \mathrm{m}^{2}$ & $\mathrm{~d} 1$ & $1 / 7 d$ \\
\hline Capecitabine & $1,000 \mathrm{mg} / \mathrm{m}^{2}$, b.i.d. & Days $1-14$ & $1 / 21 d$ \\
\hline \multicolumn{4}{|l|}{ GT } \\
\hline Gemcitabine & $1,000 \mathrm{mg} / \mathrm{m}^{2}$ & Days 1 and 8 & $1 / 21 d$ \\
\hline Paclitaxel & $175 \mathrm{mg} / \mathrm{m}^{2}$ & d1 & $1 / 21 d$ \\
\hline \multicolumn{4}{|l|}{ NX } \\
\hline Vinorelbine & $25 \mathrm{mg} / \mathrm{m}^{2}$ & Days 1 and 8 & $1 / 21 d$ \\
\hline Capecitabine & $1,000 \mathrm{mg} / \mathrm{m}^{2}$, b.i.d. & Days $1-14$ & $1 / 21 d$ \\
\hline \multicolumn{4}{|l|}{ NP } \\
\hline Vinorelbine & $25 \mathrm{mg} / \mathrm{m}^{2}$ & Days 1 and 8 & $1 / 21 d$ \\
\hline Cisplatin & $25 \mathrm{mg} / \mathrm{m}^{2}$ & Days $1-3$ & $1 / 21 d$ \\
\hline Or carboplatin & $A \cup C=2$ & Days 1 and 8 & \\
\hline \multicolumn{4}{|l|}{ GP } \\
\hline Gemcitabine & $1,000 \mathrm{mg} / \mathrm{m}^{2}$ & Days 1 and 8 & $1 / 21 d$ \\
\hline Cisplatin & $25 \mathrm{mg} / \mathrm{m}^{2}$ & Days $1-3$ & $1 / 21 d$ \\
\hline Or carboplatin & $A \cup C=2$ & Days 1 and 8 & \\
\hline \multicolumn{4}{|l|}{$\mathrm{T}+$ bevacizumab } \\
\hline Albumin paclitaxel & $100-150 \mathrm{mg} / \mathrm{m}^{2}$ & d1 & $1 / 7 d$ \\
\hline Bevacizumab & $10 \mathrm{mg} / \mathrm{kg}$ & $\mathrm{d} 1$ & $1 / 21 d$ \\
\hline \multicolumn{4}{|l|}{$\mathrm{X}+$ bevacizumab } \\
\hline Capecitabine & $1,000 \mathrm{mg} / \mathrm{m}^{2}$, b.i.d. & Days $1-14$ & $1 / 21 d$ \\
\hline Bevacizumab & $10 \mathrm{mg} / \mathrm{kg}$ & $\mathrm{d} 1$ & $1 / 21 d$ \\
\hline
\end{tabular}


Usage and dosage of endocrine drugs for breast cancer:

(I) Tamoxifen citrate: $10 \mathrm{mg}$ orally bid.

Toremifene citrate: $60 \mathrm{mg}$ orally qd.

(II) Aromatase inhibitors (AI)

Anastrozole: $1 \mathrm{mg}$ orally qd.

Letrozole: $2.5 \mathrm{mg}$ orally qd.

Exemestane: $25 \mathrm{mg}$ orally qd.

(III) Fulvestrant: $500 \mathrm{mg}$ IM every 4 weeks.

(IV) CDK4/6 inhibitors.

Palbociclib: $125 \mathrm{mg}$ orally qd for 21 days and then wait for 7 days.

(V) HDAC inhibitors.

Chidamide: $30 \mathrm{mg}$ orally, 2 times a week (the interval between two doses should not be less than 3 days; for instance, on Monday and Thursday).

(VI) Progesterone: medroxyprogesterone: $0.5 \mathrm{~g}$ orally bid.

\section{Anti-bone metastasis therapy}

Usage of bone-modifying agents for breast cancer bone metastasis:

(I) Zoledronic acid $4 \mathrm{mg}$, intravenous infusion for $>15$ minutes, once every 3 to 4 weeks. For patients with stable disease after bone metastases, the dosing frequency can be changed to once every 3 months after 2 years.

(II) Ibandronic acid $6 \mathrm{mg}$, intravenous infusion for $>2$ hours, once every 3 to 4 weeks. Loading dose of ibandronic acid: For patients with severe pain and urgent need to improve their quality of life, a loading dose of ibandronic acid: $6 \mathrm{mg} / \mathrm{d}$, intravenously infused for 3 consecutive days, and then once every 3 to 4 weeks for routine use.

(III) Desulumab $120 \mathrm{mg}$, subcutaneous injection, once every 4 weeks.

(IV) Pamidronate disodium 60-90 mg, intravenous infusion for $>2$ hours, once every 3-4 weeks.

(V) Clodronate disodium $400 \mathrm{mg} / \mathrm{d}$, intravenous infusion for 3 consecutive days, followed by oral 1,600 mg/d; each cycle is 3-4 weeks long.

Notes: ${ }^{1}$ The definition of skeletal-related events (SREs) in clinical studies: SREs include intensified bone pain or new bone pain, pathological fractures (vertebral fractures or non-vertebral fractures), vertebral compression or deformation, spinal cord compression, symptoms following bone radiotherapy (for bone pain or for the prevention/treatment of pathological fractures or spinal cord compression), and hypercalcemia. SREs such as bone pain and bone injury are common symptoms of breast cancer bone metastasis and can seriously undermine quality of life. SREs are the main factors affecting the patient's ability to move independently and the quality of life. Among them, spinal cord compression is a cancer-related emergency requiring multidisciplinary consultations (including the inputs of orthopedic experts). Corticosteroids and other treatments for dehydration should be applied to promptly relieve the compression and reduce the limb dysfunction and even paraplegia caused by spinal cord compression.

${ }^{2}$ Emission computed tomography (ECT) of bones is the most commonly used method for screening bone metastasis. Bone ECT is recommended for the routine screening of breast cancer patients with suspicious bone metastasis symptoms including bone pain, pathologic fracture, increased alkaline phosphatase level, and hypercalcemia. It can also be used for routine examinations in patients with locally advanced breast cancer (above T3N1M0) and/or recurrent/metastatic breast cancer. When bone ECT is used for evaluating treatment response, an increasing number of sites with increased tracer uptake does not necessarily mean disease progression. Bone-window CT scan should be performed. If the original osteolytic lesions have been transformed into bone calcification and the new sites with increased tracer uptake also have the appearance of bone calcification, it can be assessed as effective. If the new sites with increased tracer uptake exhibit the appearance of osteolytic lesions, it can be assessed as disease progress.

${ }^{3}$ Bone CT and X-ray examinations are the main imaging methods for clinical diagnosis of bone metastases. In patients with abnormal ECT findings, MRI, CT (bone window), and X-ray should be performed in sites with suspicious bone metastatic lesions, so as to confirm the possible bone metastasis and learn the severity of bone destruction. 
${ }^{4}$ Bone biopsy is an invasive examination. When the imaging findings are inconsistent with the clinical findings, it is recommended to perform bone biopsy on the suspected area to determine whether the affected bone is the result of a bone metastasis. Bone biopsy should also be considered in patients with a single suspicious lesion.

${ }^{5}$ PET-CT has similar similar sensitivity with bone ECT. However, the expert panel believes that the value of PET-CT in diagnosing bone metastasis still requires further investigations. Currently it is not routinely recommended in clinical settings. ${ }^{6}$ Bone metastases of breast cancer are frequently seen as multiple osteolytic lesions. In some patients, the repaired osteolytic lesions after treatment may be radiologically seen as excessive calcification and thus misdiagnosed as osteogenic changes. In these patients, the X-ray or CT film at the first presentation should be reviewed to identify the presence of any osteolytic lesion. A diagnosis of bone metastases cannot be made if there is only abnormal bone ECT finding and/or abnormally elevated alkaline phosphatase or lactate dehydrogenase but without abnormal findings on MRI, CT, or X-ray. Reexaminations with bone ECT or bone CT within 3 months is recommended. If there are more site with increased tracer uptake, further examinations should be performed to confirm the diagnosis. For efficacy assessment of bone metastasis, a multidisciplinary analysis is required based on clinical symptoms, tumor markers, and imaging changes. Avoid a subjective judgment based on symptoms only; also, avoid make a decision based on the imaging findings only without inquiring about pain symptoms and change in quality of life.

${ }^{7}$ The treatment of breast cancer bone metastasis should be based on systemic therapies including chemotherapy, endocrine therapy, and molecularly targeted therapy. Factors affecting the choice of systemic treatment include the hormone receptor status and HER2 status of a breast cancer, patient's age and menstrual status, and whether the disease progresses slowly. Breast cancer bone metastasis generally is not directly life-threatening, and breast cancer bone metastasis patients without visceral metastasis usually have relatively long survival; thus, endocrine therapy is preferred in patients with hormone receptorpositive tumors that progress relatively slowly and without primary endocrine resistance. For patients with ER- and PRnegative tumors, short postoperative disease-free interval, rapid disease progression, or HR-positive tumors and with primary endocrine resistance, single-agent chemotherapy is preferred in patients with a single bone metastasis or asymptomatic visceral metastasis, whereas combination chemotherapy should only be considered for patients with bone metastases requiring rapid symptom control or those with symptomatic visceral metastases. For patients with HER-2-positive bone metastases, the treatment principle is the same as that for patients with metastases to other sites-combination with an HER-2-targeted therapy is preferred.

${ }^{8}$ Administration advice: (I) serum electrolyte levels, particularly serum creatinine, calcium, phosphate, and magnesium levels, should be measured before the use of bisphosphonates. (II) Calcium and vitamin D should be supplemented on a daily basis after long-term use of bisphosphonate combinations (calcium: 1,200-1,500 mg/d; Vitamin D3: 400-800 IU). (III) No dose adjustment is needed in patients with mild or moderate renal insufficiency (creatinine clearance larger than $30 \mathrm{~mL} / \mathrm{min}$ ). However, for patients with severe renal insufficiency (creatinine clearance less than or equal to $30 \mathrm{~mL} / \mathrm{min}$ ), the dose should be adjusted or the duration of infusion should be prolonged in accordance with different product specifications. Patients with creatinine clearance less than $30 \mathrm{~mL} / \mathrm{min}$ or on dialysis should be closely monitored during treatment with desulumab to avoid the occurrence of hypocalcemia. (IV) It has been reported that bisphosphonate-associated osteonecrosis of the jaw $(\mathrm{BONJ})$ is a morbid bone disease linked to long-term bisphosphonate use. Therefore, oral examinations and appropriate prophylactic treatments should be performed before using bisphosphonates. During medication, attention should be paid to oral hygiene, and oral surgeries such as tooth extraction should be avoided as possible. If there are persisting areas of exposed bone in the maxillofacial region that occur without a known reason during medication or after a surgery, consult a specialist as soon as possible.

${ }^{9}$ Indications for discontinuation of therapy. (I) Adverse reactions are detected during drug administration and they are clearly related to the use of bone-modifying agents. (II) The tumor progresses during the treatment as it spreads to other organs and can be life-threatening. (III) The clinicians believe it is necessary to stop the medications. (IV) Relief of bone pain after treatment is not an indication for discontinuation of therapy.

${ }^{10}$ Surgical treatment of bone metastasis. The purposes of surgical treatment are to improve the patients' quality of life by resolving nerve compression, alleviating pain, and restoring limb functions. Patients with bone metastasis must be closely followed up. A proper decision-making on the surgery on the long bones with potentially pathological fractures is critically important to improve the patients' quality of life if an effective surgical treatment is performed before fracture and/or before 
spinal cord compression. Surgical procedures for breast cancer bone metastases include simple internal fixation, debridement plus internal fixation, lesion resection plus artificial joint replacement, decompression after spinal cord compression, and reconstruction for increasing spinal stability. Fixation can be selectively applied for pathological fracture or spinal cord compression, especially in breast cancer bone metastasis patients with an expected survival time of $>3$ weeks. Prophylactic fixation can be selectively applied in breast cancer bone metastasis patients with one or more of the following conditions: femoral metastasis sized $>2.5 \mathrm{~cm}$; metastasis to the neck of femur bone; bone cortical destruction $>5 \%$; and/or with an expected survival time of $>3$ months. After the bone metastases have been controlled with multidisciplinary treatments, orthopedists should be timely consulted to determine the timing of surgery.

${ }^{11}$ Radiotherapy for bone metastasis. The main role of radiotherapy in patients with breast cancer bone metastasis is to relieve bone pain and reduce the risk of pathological fractures. Radiotherapy includes external-beam irradiation and radionuclide therapy. External-beam irradiation is a common effective method during the palliative therapy for bone metastases. The main indications of external-beam irradiation include: symptomatic bone metastases, for which the treatment is to alleviate pain and restore functions; and, selectively used for the prophylactic radiotherapy of metastasis to weight-bearing bones (e.g., spine and femur). The doses of external-beam irradiation include $30 \mathrm{~Gy} / 10$ fractions, $20 \mathrm{~Gy} / 5$ fractions, and $8 \mathrm{~Gy} / \mathrm{fraction}$. Although these doses have comparable effectiveness in stopping acute analgesia, long-course radiotherapy can achieve longer pain control. Clinically, appropriate radiotherapy fractionation should be selected according to the expected survival time and the tolerance dose of normal tissues related to the metastases. Effective external-beam irradiation can achieve objective pain relief in $2 / 3$ of patients and achieve complete pain relief in $1 / 3$ of patients. For patients who are eligible for stereotactic body radiation therapy (SBRT), the irradiation dose can be increased and the normal tissues can be better protected. Radionuclide therapy is useful in easing diffuse pain due to bone metastasis; however, in some patients, radionuclide therapy may result in high incidence of bone marrow suppression, which can only be restored slowly (about 12 weeks) and thus affect the implementation of chemotherapy. Therefore, the clinical use of radionuclide therapy should only be performed in carefully selected cases at the right time.

\section{Management of therapy-induced disorders}

\section{Management of chemotherapy-induced disorders: Nausea and vomiting}

(I) Among the adverse reactions of systemic chemotherapy, chemotherapy-induced nausea and vomiting (CINV) is one of the most dreaded side effects of cancer therapy and can dramatically affect subsequent chemotherapy. In severe cases, CINV can lead to electrolyte imbalance and metabolic alkalosis and affect the dosage and course of chemotherapy. Some patients may have to stop chemotherapy due to CINV. Therefore, antiemetic control is particularly important during chemotherapy. Antiemetic prophylaxis should be routinely applied to ensure the smooth implementation of chemotherapy.

(II) Grading of CINV

(i) Grading of intravenous chemotherapy-induced nausea and vomiting.

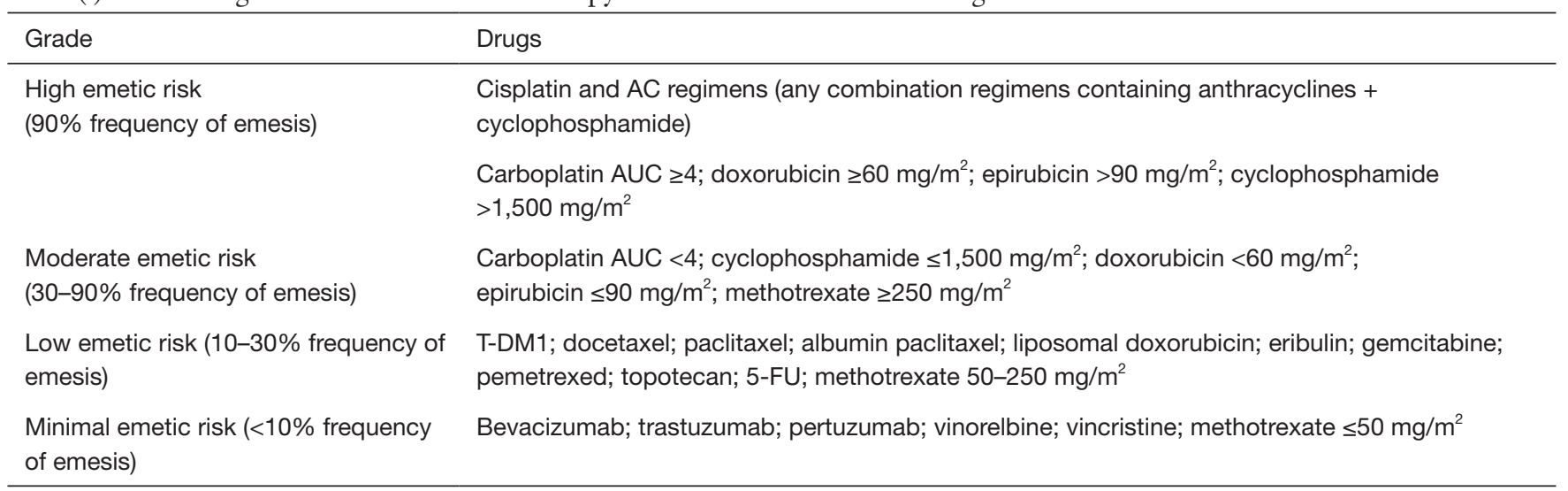


(ii) Oral anticancer drugs with moderate to high emetic risk: Cyclophosphamide $\left[\geq 100 \mathrm{mg} /\left(\mathrm{m}^{2} \bullet \mathrm{d}\right)\right]$, etoposide, olaparib, and temozolomide $\left[>75 \mathrm{mg} /\left(\mathrm{m}^{2} \bullet \mathrm{d}\right)\right]$.

(III) Prevention of acute and delayed vomiting during chemotherapy

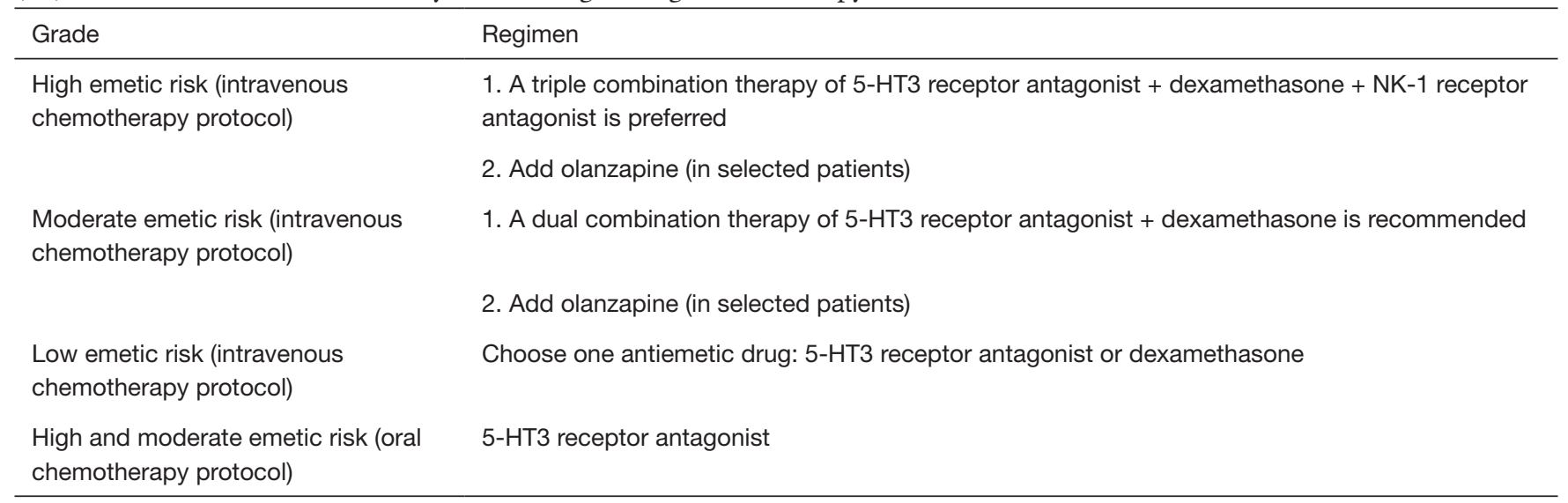

Usage and dosage of drugs in chemotherapy regimens:

(I) Intravenous chemotherapy with high emetic risk (a triple combination therapy of 5-HT3 receptor antagonist + dexamethasone + NK-1 receptor antagonist).

Before chemotherapy:

(i) Any of the following 5-HT3 receptor antagonists: ondansetron, granisetron, and palonosetron [Note: multiple dosage forms including injections, oral-dosage forms, and transdermal patches (granisetron) have been available and may be selected according to the specific needs of the patients].

(ii) Aprepitant day 1: $125 \mathrm{mg}$, days 2 and 3: $80 \mathrm{mg}$, oral-dosage.

(iii) Patients on AC regimen: dexamethasone day 1: 6-12 mg orally and intravenously administered; cisplatin regimen and patients at risk of delayed vomiting; dexamethasone day 1: $6 \mathrm{mg}$ oral-dosage, day 2-4: $3.75 \mathrm{mg}$, orally administered.

(II) Intravenous chemotherapy with moderate emetic risk (a dual combination therapy of 5-HT3 receptor antagonist + dexamethasone).

Before chemotherapy:

(i) Any of the following 5-HT3 receptor antagonists: ondansetron, granisetron, and palonosetron [Note: multiple dosage forms including injections, oral-dosage forms, and transdermal patches (granisetron) have been available and may be selected according to the specific needs of the patients].

(ii) Dexamethasone: 6-12 mg, orally/intravenously administered.

(III) Intravenous chemotherapy with low emetic risk (5-HT3 receptor antagonist or dexamethasone).

Before chemotherapy:

(i) Any of the following 5-HT3 receptor antagonists: ondansetron, granisetron, and palonosetron [Note: multiple dosage forms including injections, oral-dosage forms, and transdermal patches (granisetron) have been available and may be selected according to the specific needs of the patients].

(ii) Dexamethasone: $6-12 \mathrm{mg}$, orally/intravenously administered.

(IV) Oral chemotherapy with high and moderate emetic risk (5-HT3 receptor antagonist).

During oral chemotherapy: 5-HT3 receptor antagonists are continuously administered on a daily bais, and oral or topical dosage forms are recommended.

Notes: ${ }^{1}$ Olanzapine, a psychotropic drug, can relieve anxiety and depression in cancer patients. For patients with fulminant or refractory vomiting after using the standard triple combination regimen and for patients with emotional distress (anxiety and depression), the addition of olanzapine at a dose of 5-10 $\mathrm{mg}$ qd (Level of Evidence: A).

${ }^{2}$ The dosage of dexamethasone follows the principle of individualized treatment. Treatment tolerance and side effects of glucocorticoids should be assessed. Avoid using dexamethasone in patients treated with immune checkpoint inhibitors. 
${ }^{3}$ Aprepitant may increase the exposure level of dexamethasone through CYP3A4. Therefore, when used in combination with aprepitant (125 mg/80 mg therapy), dexamethasone should be used at a lower dose (about half of the conventional dose). Special attention should also be paid to the metabolism changes and the relevant effects caused by the interaction between aprepitant and other drugs (see the drug labels for details).

\section{Management of chemotherapy-induced disorders: bone marrow suppression}

(I) Bone marrow suppression is a common non-specific toxicity of chemotherapy, and it is also a key factor affecting the course and dose of chemotherapy. In most cases, the white blood cell (WBC) count decreases 1-2 weeks after chemotherapy is administered, reaches the lowest level 10-14 days after chemotherapy, and returns normal after 3-4 weeks.

(II) Risk grading and primary preventive measures of febrile neutropenia (FN) caused by breast cancer chemotherapy:

\begin{tabular}{|c|c|c|}
\hline Risk grading & Chemotherapy regimen & Preventive treatment \\
\hline $\begin{array}{l}\text { Moderate risk } \\
\text { FN probability of } 10-20 \%\end{array}$ & $\begin{array}{l}\text { AC (doxorubicin + cyclophosphamide) } \\
A C \rightarrow T \pm H P \text { (doxorubicin }+ \text { cyclophosphamide sequential } \\
\text { docetaxel } \pm \text { trastuzumab + pertuzumab) }\end{array}$ & $\begin{array}{l}\text { Based on the risk factors: } \\
\text { prophylactic application of G-CSF } \\
\text { is considered }\end{array}$ \\
\hline & $\begin{array}{l}\text { FEC } \rightarrow \text { T (fluorouracil + epirubicin + cyclophosphamide sequential docetaxel) } \\
\text { 3-weekly docetaxel } \\
\text { 3- and 2-weekly paclitaxel } \\
\text { TH (weekly paclitaxel + trastuzumab) }\end{array}$ & \\
\hline
\end{tabular}

Low risk

FN probability of $<10 \%$
Prophylactic application of G-CSF

is not recommended

Note: ${ }^{1} \mathrm{FN}$ refers to significant neutrophil reduction accompanied by fever, usually defined as an absolute neutrophil count (ANC) of $<0.5 \times 10^{9} / \mathrm{L}$, or $<1.0 \times 10^{9} / \mathrm{L}$ predicted to fall below $0.5 \times 10^{9} / \mathrm{L}$ within 48 hours, with the presence of fever (a single measurement of oral temperature $\geq 38.5$ or $\geq 38.0^{\circ} \mathrm{C}$ for more than 1 hour, or axillary temperature $>38.5^{\circ} \mathrm{C}$ for more than 1 hour).

${ }^{2}$ Granulocyte colony stimulating factor (G-CSF) mainly includes recombinant human G-CSF (rhG-CSF) and polyethylene glycol recombinant human G-CSF (PEG-rhG-CSF).

${ }^{3}$ The risk of FN should be assessed before chemotherapy, and corresponding preventive measures should be taken according to the chemotherapy regimen, dose intensity, patient's risk factors, and treatment objectives. (I) For patients receiving chemotherapy regimens with moderate or high risk of FN, prophylactic use of G-CSF should be considered no matter the chemotherapy is performed for curing the disease, prolonging survival, or improving disease-related symptoms. (II) For patients receiving chemotherapy regimens with low risk of FN, routine use of G-CSF is not recommended. However, if FN or dose-limited neutropenia (or agranulocytosis) occurs in the first chemotherapy cycle, prophylactic use of G-CSF may be considered in the next cycle (secondary prevention). (III) Considering the efficacy and convenience of prophylactic use of PEG-rhG-CSF, the experts recommend that long-acting preparations are preferred in high-risk patients. Dose of prophylactic use of PEG-rhG-CSF: body weight $>45 \mathrm{~kg}$ : $6 \mathrm{mg}$ per cycle; body weight $\leq 45 \mathrm{~kg}$ : $3 \mathrm{mg}$ per cycle. Begin 24 to 72 hours after completion of chemotherapy. For patients with excessively fast increase in granulocyte count after the first cycle of application, the dose can be reduced to $3 \mathrm{mg}$ in the subsequent cycles; if the drug used is rhG-CSF, the dose can be $2 \mu \mathrm{g} / \mathrm{kg} \mathrm{qd}$, which should be administered 3-4 days after chemotherapy until ANC returns to normal or close to normal level (laboratory standard).

\section{Chemotherapy management: monitoring and prevention of cardiotoxicity}

(I) The cardiotoxicity of breast cancer chemotherapy mainly comes from anthracyclines, and the cardiotoxicity of 
anthracyclines is commonly progressive and irreversible. Use of anthracyclines for the first time can cause damage to the heart, which is cumulative and can affect anti-tumor treatment and undermine the quality of life.

(II) Maximum cumulative lifetime dosages of common anthracyclines and anthraquinones

\begin{tabular}{ll}
\hline Drug name & Recommended maximum cumulative lifetime dosages \\
\hline Adriamycin & $550 \mathrm{mg} / \mathrm{m}^{2}\left(<350-400 \mathrm{mg} / \mathrm{m}^{2}\right.$ for radiotherapy or combined therapy $)$ \\
Epirubicin & $900-1,000 \mathrm{mg} / \mathrm{m}^{2}$ \\
Pirarubicin & $950 \mathrm{mg} / \mathrm{m}^{2}$ \\
\hline
\end{tabular}

(III) Monitoring and evaluation of cardiotoxicity

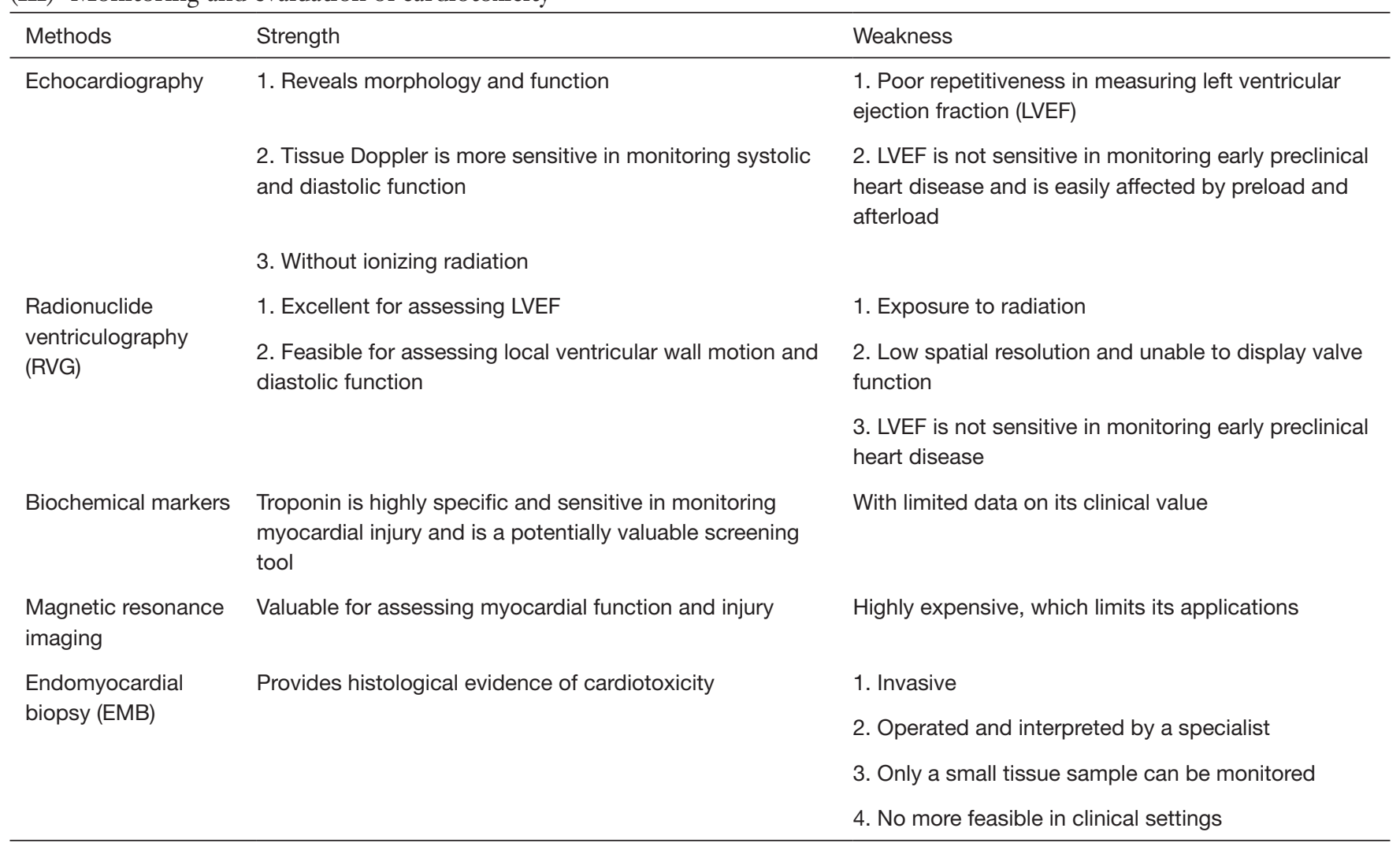

(IV) Prevention of cardiotoxicity after chemotherapy

(i) The risk factors of cardiac injuries include previous history of cardiovascular disease, previous application of anthracycline chemotherapy or radiotherapy, and aged older than 65 years old. For patients with any of the above risk factors, the risk of cardiotoxicity should be thoroughly assessed before medication, and the regimen and dose should be adjusted accordingly. Use of anthracyclines should be avoided in high-risk patients. Early monitoring and prevention of cardiotoxicity is required for breast cancer patients receiving anthracyclines. For patients whose LVEF has been reduced by more than $10 \%$, more sensitive methods (e.g., dynamic monitoring of troponin) should be applied.

(ii) Prophylaxis: dexrazoxane is recommended for patients receiving anthracyclines for the first use to effectively prevent anthracycline-induced cardiotoxicity (1A). The dose ratio of dexrazoxane to anthracyclines is 10:1 or 20:1. Anthracyclines should be administered immediately after completing the infusion of dexrazoxane. Other cardioprotective agents including coenzyme Q10, N-acetylcysteine, antioxidants (e.g., vitamins $\mathrm{C}$ and $\mathrm{E}$ ), and iron chelating agents may also have beneficial effects on the heart and brain, but their roles in preventing and treating anthracycline-induced cardiotoxicity warrant further investigations.

(iii) The chronic and delayed anthracycline-induced cardiotoxicity is related to the total dose administered. Therefore, 
limiting the cumulative dose of anthracyclines can lower the incidence of cardiotoxicity. Liposome anthracyclines (e.g., liposomal adriamycin and liposomal daunorubicin) may reduce the incidence of anthracycline-induced cardiotoxicity.

(iv) When cardiac symptoms occur, consult a cardiologist to offer symptomatic treatments.

\section{Endocrine therapy: drug tolerability and medication precautions}

(I) Precautions for TAM medication.

(i) The serious adverse reactions of TAM include venous thrombosis and endometrial cancer. Long-term drug use, postmenopausal state, and irregular vaginal bleeding are associated with increased risk of endometrial disease.

(ii) Gynecological examination should be performed every 12 months during the use of TAM, and the frequency of monitoring can be increased as appropriate if any of the above risk factors is present.

(iii) Endometrial biopsy is recommended for postmenopausal patients with thickened endometrium (thickness $>8 \mathrm{~mm}$ ). When the endometrial thickness ranges $5-8 \mathrm{~mm}$, a biopsy procedure should be performed (or not) should also be based on the clinical manifestations. Endometrial thickness is not an indication for biopsy in premenopausal patients.

(II) Precautions for AI medication

(i) Long-term AI use may cause osteoporosis, joint pain, and other adverse reactions.

(ii) Bone mineral density should be routinely monitored before (at baseline) and during the administration of medications. It is recommended to do it every 6 months, and the interval should be no longer than 1 year. A BMD T-score can be obtained. A T-score of $<-2.5$ indicates osteoporosis, which requires bisphosphonate therapy; a T-score between -1.5 and -1.0 reveals bone loss, which can be treated with vitamin $\mathrm{D}$ and calcium tablets, and use of bisphosphonates may be considered; a T-score of >-1.0 means normal bone mass, and bisphosphonate therapy is not recommended. Bisphosphonates can be administered once every 3 to 6 months. Dental examinations should be performed before bisphosphonate treatment.

(III) Breast cancer patients may experience decreased ovarian function due to physiological and therapeutic factors, leading to menopausal-related symptoms, urogenital symptoms, and low bone mass/osteoporosis.

(i) Breast cancer is a contraindication for hormone replacement therapy (HRT). Many other non-hormonal preparations including botanicals (e.g., isopropanolic black cohosh extract), phytoestrogen, traditional Chinese medicine, or selective serotonin reuptake inhibitors can be selected to treat menopausal symptoms.

(ii) Non-hormonal therapy is the treatment of choice for local genitourinary symptoms, and the benefits and risks of vaginal estrogen must be fully assessed before its use.

\section{Cardiotoxicity of trastuzumab and its management}

(I) Cardiotoxicity of trastuzumab.

Trastuzumab combined with chemotherapy drugs, especially anthracyclines, can aggravate myocardial injury and may even lead to heart failure. Therefore, trastuzumab combined with anthracycline chemotherapy is not recommended for patients with recurrent and metastatic breast cancer. Adjuvant trastuzumab should be applied after anthracycline chemotherapy. Neoadjuvant trastuzumab can be used under close monitoring, during which trastuzumab should be given concurrently with short-course anthracycline chemotherapy (within 4 cycles).

(II) Monitoring of trastuzumab-associated cardiotoxicity.

Trastuzumab-related cardiotoxicity was not frequent in clinical studies and in most cases it could be reversed, which might be explained that subjects enrolled in clinical studies had been screened for cardiac safety after chemotherapy. Therefore, in clinical practice, baseline assessments including history taking, physical examination, electrocardiography, and echocardiography (e.g., for LVEF) should be performed before the commencement of trastuzumab treatment, and cardiac function should be monitored every 3 months during trastuzumab administration. The frequency of monitoring should be even higher (e.g., once every 6 to 8 weeks) in patients with asymptomatic cardiac insufficiency. 
(III) Suspension, resumption, and permanent discontinuation of trastuzumab.

\begin{tabular}{ll}
$\begin{array}{l}\text { Trastuzumab treatment can be suspended for at } \\
\text { least } 4 \text { weeks, with LVEF measured every } 4 \text { weeks }\end{array}$ & $\begin{array}{l}\text { Trastuzumab should be withheld for four weeks if the LVEF decreases } \geq 16 \% \text { from } \\
\text { preuse values or decreases below normal and } \geq 10 \% \text { absolute decrease in LVEF } \\
\text { from preuse values }\end{array}$ \\
Resumption of trastuzumab use & LVEF normalizes or the absolute decrease from baseline is $\leq 15 \%$ within $4-8$ weeks \\
Permanent discontinuation of trastuzumab & $\begin{array}{l}\text { Decline in LVEF that persists for }>8 \text { weeks, or has had the drug held on more than } 3 \\
\text { occasions due to cardiac problems }\end{array}$ \\
\hline
\end{tabular}

Notes: ${ }^{1}$ The risk of brain metastasis is rising in patient with advanced breast cancer, mainly due to the following two reasons: (I) the availability of more effective systemic treatment of breast cancer has prolonged the survival of patients; and (II) the application of brain MRI has found more patients with asymptomatic brain metastases. The incidence of brain metastasis differs in patients with different types of breast cancer. Generally, the risk of brain metastasis is relatively high in patients with triple-negative breast cancer or HER-2-positive breast cancer, suggesting the importance of monitoring the occurrence of brain metastasis in these patients. In addition, studies have shown that high histological grade and high proliferative activity of the primary tumor, young age, high tumor burden, and BRCA gene mutations are also high-risk factors for brain metastasis. The most common site of brain metastases is the cerebrum. Less often, cancer spreads to the cerebellum and brain stem.

2"A limited number of brain metastases" generally means that the number of metastases is not necessarily limited to 3 or less, but the maximum diameter of a single lesion should not exceed $3 \mathrm{~cm}$; all the lesions can be treated with SRT, and the local control rates are consistent with that of whole-brain radiotherapy.

${ }^{3}$ For patients with recurrence after local treatment of brain metastases, re-operation or SRT may be considered if there is no history of intracranial radiotherapy, the general condition is good, and the extracranial lesions are well controlled. Wholebrain radiotherapy (SRT with hippocampal protection) may also be considered, along with the use of memantine. If the size of the metastasis exceeds the indications of SRT and is not suitable for re-operation, whole-brain radiotherapy can be considered. For patients experience relapse after whole-brain radiotherapy, SRT can be considered. If relapse occurs after SRT, SRT or whole-brain radiotherapy can be repeated. In short, the treatment strategy for recurrence after local treatment of brain metastases should consider the patient's physical condition, the control of extracranial lesions, the patient's quality of life, and the possible benefit from treatment.

${ }^{4}$ Many studies have confirmed that less than $10 \%$ of patients presented with brain metastases within $5 \mathrm{~mm}$ of the hippocampus and no patient presented with a metastasis in the hippocampus itself. Whole-brain radiotherapy with hippocampal avoidance, combined with memantine if appropriate, is recommended for patients with good systemic condition, satisfactory control of extracranial lesions, and a distance of not less than $1 \mathrm{~cm}$ between the lesion and the hippocampus, because such a protocol has high efficiency and low toxicity.

${ }^{5}$ Generally, the effectiveness of medications is far from satisfactory in treating breast cancer brain metastasis. Studies have shown that chemotherapy drugs including capecitabine, topotecan, and temozolomide may achieve certain responses in patients with brain metastases. Other anti-HER-2 small-molecule tyrosine kinase inhibitors such as pyrotinib have also shown certain activities against brain metastases.

${ }^{6}$ Symptomatic and supportive treatment is one of the main treatments for breast cancer brain metastases as it can improve the quality of life of patients and help the implementation of radiotherapy and drug treatment. For patients with intracranial hypertension, mannitol, glucocorticoids (e.g., dexamethasone), diuretics, and other drugs should be routinely used to alleviate the symptoms of cerebral edema. For patients with intractable brain edema after radiotherapy, bevacizumab may be given to attenuate brain edema. Patients with epilepsy should be treated with antiepileptic drugs. 